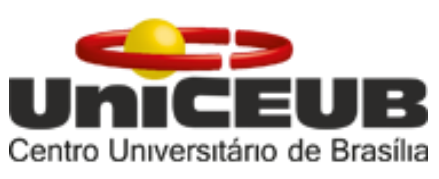

CENTRO UNIVERSITÁRIO DE BRASÍLIA - UniCEUB FACULDADE DE CIÊNCIAS DA EDUCAÇÃO E DA SAÚDE - FACES

PROGRAMA DE INICIAÇÃO CIENTÍFICA

\author{
LUCAS FERREIRA DE CASTRO \\ MARCELLA MATEUS CAVALCANTE
}

CONDUTA EXPECTANTE (NÃO CIRURGICA) EM PACIENTES PEDIÁTRICOS VÍTIMAS DE TRAUMA ABDOMINAL CONTUSO

COM LESÃO DE BAÇO E/OU FÍGADO.

BRASÍLIA

2017 


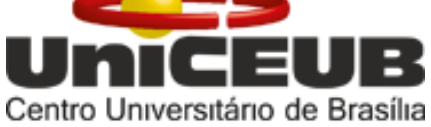

LUCAS FERREIRA DE CASTRO

MARCELLA MATEUS CAVALCANTE

\section{CONDUTA EXPECTANTE (NÃO CIRURGICA) EM PACIENTES PEDIÁTRICOS VÍTIMAS DE TRAUMA ABDOMINAL CONTUSO COM LESÃO DE BAÇO E/OU FíGADO}

\begin{abstract}
Relatório final de pesquisa de Iniciação Científica apresentado à Assessoria de Pós-Graduação e Pesquisa pela Faculdade de Ciências da Educação e da Saúde - FACES.
\end{abstract}

Orientação: Prof.Mํㅗ Andréa Lopes

Ramires Kairala.

\section{BRASÍLIA}




\title{
CONDUTA EXPECTANTE (NÃO CIRURGICA) EM PACIENTES PEDIÁTRICOS \\ VÍTIMAS DE TRAUMA ABDOMINAL CONTUSO \\ COM LESÃO DE BAÇO E/OU FÍGADO
}

\author{
Lucas Ferreira de Castro - UniCEUB, PIC Institucional, aluno voluntário \\ lucas.f.c94@gmail.com
}

Marcella Mateus Cavalcante - UniCEUB, PIC institucional, aluno voluntário marcella.mateus@gmail.com

\section{Andréa Lopes Ramires Kairala - UniCEUB, professora orientadora kairalak@uol.com.br}

O trauma abdominal contuso e seu impacto na morbidade e mortalidade de pacientes pediátricos exige condutas eficazes para reduzir riscos e aumentar as taxas de sobrevida com qualidade. Nesse contexto, as condutas não-cirúrgicas têm grande relevância nos cenários de trauma, com menores índices de complicações associadas aos atos cirúrgicos. $O$ objetivo desse estudo foi analisar o perfil de pacientes e os fatores relacionados a falha do tratamento não cirúrgico em pacientes com lesão esplênica e/ou hepática após trauma abdominal fechado internados em um hospital de referência para trauma no DF.Estudo quantitativo, descritivo e retrospectivo com análise de prontuários de crianças entre 0 e 16 anos atendidas em hospital terciário pela equipe de cirurgia pediátrica, entre os anos de 2012 e 2016, vítimas de trauma abdominal contuso com lesão hepática e/ou esplênica e que foram submetidos a tratamento expectante(CE) ou não cirúrgico. As complicações, tempo de permanência hospitalar e o índice de trauma (RTS, ISS e TRISS) foram analisados. Critérios de exclusão: óbito nas primeiras 6 horas de internação, alta hospitalar em menos de 24 horas, paciente com lesão de vísceras ocas associadas e prontuários incompletos.Foram analisados inicialmente 312 prontuários; 65 entraram nos critérios de inclusão, dentre os quais $13,8 \%$ foram submetidos a conduta cirúrgica inicial e $86,1 \%$ a CE. Com relação ao ano de atendimento: $2012=20 \%$; $2013=21 \%, 2014=37 \%, 2015=11 \%$ e $2016=11 \%$. Quanto a víscera lesada, 50\% dos pacientes apresentaram lesão 
hepática, $42 \%$ esplênica e $8 \%(\mathrm{~N}=5)$ de ambas as vísceras. As lesões hepáticas foram relacionadas com estadiamento de gravidade mais leve e lesões esplênicas com lesões mais graves ( $p$-valor $=0,048)$. Foram a óbito $6 \%(\mathrm{~N}=4)$ dos pacientes.Predomínio de pacientes do sexo masculino $65 \%(\mathrm{~N}=42)$ e pacientes entre 6 e 10 anos, 48,4\%.Escolares apresentaram maior proporção de lesão esplênica e pré escolares lesões hepáticas ( $p$-valor=0,002). As principais causas de lesão foram colisão automobilística $40 \%$; atropelamento $21 \%$, queda de bicicleta $11 \%$ e $10 \%$ vítimas de acidentes domésticos. O transporte do local do acidente para o local de atendimento foi por ambulância comum em $32 \%(\mathrm{~N}=21)$, SAMU $27,7 \%(\mathrm{~N}=18)$ e $28 \%$ dos prontuários não possuía essa informação. Foram internados em UTI 60\% dos pacientes, por períodos variáveis entre 1 e 211 dias, com média de 15,9 dias. Comorbidades associadas como TCE e lesão pulmonar apresentaram maior gravidade e foram internados em UTI ( $p$ valor $=0,0022$ ). Houve maior frequência de cirurgia antes de 12 horas da admissão naqueles pacientes com hematócrito baixo ( $p$-valor $=0,015)$. Relação forte entre pacientes que necessitaram de transfusão sanguínea e internação na UTI, 85\% dos pacientes ( $p$-valor $=0,012$ ). Dos pacientes submetidos a conduta expectante, $53,57 \%$ foram internados na UTI, com percentual de óbitos de $0 \%$, entre os que não foram acompanhados na UTI, o percentual de óbitos foi de 7,7\%. A mudança da terapêutica operatória para a não-operatória (TNO), é uma opção segura, no trauma abdominal contuso de pacientes pediátricos com lesões de vísceras parenquimatosas (fígado e baço), desde que seja indicado mediante critérios técnicos explicitados. Oferece grandes vantagens, incluindo menor número de óbitos, menor riscos de infecções associados à conduta cirúrgica e menos gastos com cuidados de saúde.

Palavras-Chave: Traumatismo abdominal fechado. Tratamento não operatório. Fígado; lesões. Baço; lesões. Pediatria 


\section{SUMÁRIO}

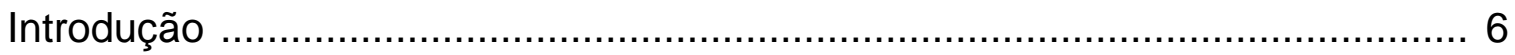

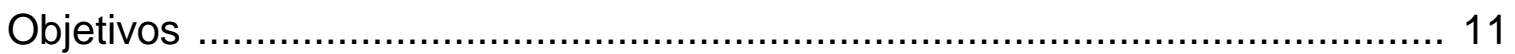

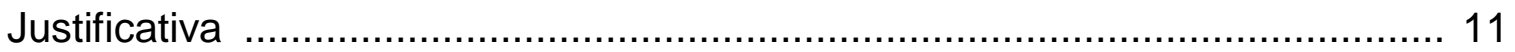

Fundamentação teórica ........................................................................ 12

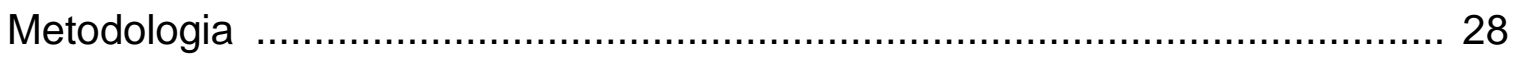

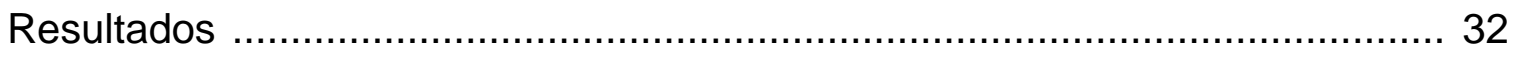

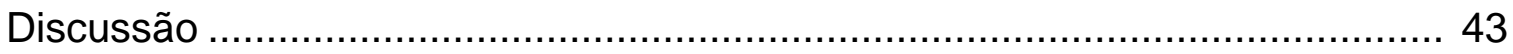

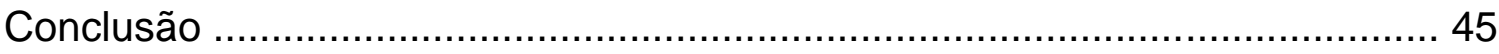

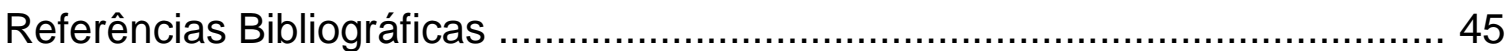




\section{INTRODUÇÃO}

Mundialmente, o trauma é a primeira causa de morte e incapacidade em crianças nos Estados Unidos. O trauma abdominal contuso é a terceira causa mais comum de óbito nos traumas pediátricos. O baço e o fígado são os órgãos mais frequentemente lesados, seguido pelo rim, intestino delgado e pâncreas. Os mecanismos mais comuns de lesão são as colisões por veículos automotivos, os acidentes de pedestres atingidos por veículos, confrontos de carros vs. bicicleta, lesões em esportes, quedas e os traumas não acidentais. (GUYTHER, 2016).

As crianças têm órgãos abdominais relativamente maiores que são cercados por menos gordura e a musculatura abdominal menos desenvolvida. O fígado e 0 baço são anatomicamente mais anteriores, tornando-os mais propensos a lesões do que os mesmos órgãos em adultos. Além disso, a caixa torácica é mais compressível, permitindo uma maior transmissão de força para órgãos como os rins, fígado e baço. O corpo de uma criança é menor, produzindo uma força maior por unidade de área de superfície corporal, sendo facilmente transmitida por todo o corpo, levando a lesões mais significativas, possivelmente sem sinais externos. (GUYTHER, 2016).

No final da década de 60 e início da década de 70 surge uma nova modalidade de manuseio do trauma abdominal fechado: o tratamento não-operatório (TNO) ou conduta expectante. Resultados encorajadores obtidos inicialmente por cirurgiões pediátricos e posteriormente por cirurgiões gerais levaram a sua progressiva aceitação, a ponto de ser considerado atualmente o tratamento de escolha para o trauma abdominal com lesão de vísceras maciças nos centros de traumatologia. (CARVALHO et al. 2009).

A lesão abdominal no trauma ocorre em aproximadamente $30 \%$ dos pacientes traumatizados com 13 e $16 \%$ de lesões de baço e fígado, respectivamente. $O$ tratamento do trauma abdominal contuso tem sofrido alterações durante as últimas décadas devido ao aumento da indicação de tratamento não operatório 
(TNO) que pode chegar a $80 \%$ dos casos. A escala para lesões de órgãos da Associação Americana de Cirurgia do Trauma (AAST-OIS), se tornou um dos fatores mais importantes para esta tomada de decisão e um importante fator preditor para falhas no TNO. (FERNANDES, 2013).

O grande número de complicações geradas pelas condutas tradicionais, a recuperação mais rápida e com demanda de menos recursos financeiros associados à conduta expectante fizeram com que essa ganhasse maior destaque nos centros de atendimento de trauma em todo o mundo. Além disso, a precisão do diagnóstico de lesão visceral por TC e o reconhecimento de complicações sépticas pós-operatórias precoces e tardias têm estimulado a adoção da prática não-operatória após o trauma abdominal fechado.

O tratamento conservador no trauma abdominal fechado consiste na simples observação clínica ou angiografia, com ou sem embolização, possibilitando diferentes condutas expectantes. $\mathrm{Na}$ observação clínica deve ser feito monitorização e cuidados intensos do paciente em ambientes adequados e os mesmos devem ficar em jejum e passar por avaliações seriadas do hematócrito. Já, a angiografia é uma terapia adjuvante que tem sido muito realizada e está indicada em pacientes com lesão de alto grau ou com extravasamento no contraste no CT, ou, ainda, pacientes com persistência da taquicardia e queda do hematócrito.

Mesmo com tamanha relevância nesse novo tipo de terapia, as razões pelas quais a terapia expectante falhou em relatos presentes em estudos são muito arbitrárias e variam muito de um estudo para outro (VELMAHOS, 2003). Além disso, a TNO tem sido bem descrita e indicada em lesões de fígado e baço graus I, II e III, mas, ainda é motivo de dúvidas e controvérsias em lesões de graus maiores. A maioria dos estudos existentes sobre o assunto não retratam muito a realidade desse tipo de terapia no Brasil, tornando as informações disponíveis desatualizadas e a maioria dos estudos baseiam-se no atendimento de pacientes adultos apesar desse tipo de conduta ter sido inicialmente descrito em pacientes da pediatria. 
A maioria das injúrias de órgãos sólidos podem ser manejadas com condutas nãocirúrgicas. Dificilmente as injúrias hepáticas e esplênicas levam a um sangramento clínico importante, provavelmente devido aos seus pequenos vasos sanguíneos e a melhor resposta à vasoconstritores. As lesões hepáticas são a segunda causa mais comum de injúria no trauma abdominal contuso e, uma concentração de Aspartato Transaminase (AST) >200 U/L ou níveis de Alanina transaminase $(A L T)>125 \mathrm{U} / \mathrm{L}$ são indicativas de lesão hepática. A conduta conservadora é recomendada em pacientes hemodinamicamente estáveis e que receberam menos de $40 \mathrm{~mL} / \mathrm{kg}$ de sangue. (GUYTHER, 2016)

As injúrias esplênicas são as mais frequentes lesões abdominais, contribuindo para mais de $45 \%$ das injúrias viscerais. Nos pacientes hemodinamicamente estáveis, o manejo de rotina inclui repouso, mensurações seriadas de hematócrito e exames físicos seriados. A Associação Americana de Cirurgia Pediátrica recomenda a conduta expectante para lesões esplênicas e hepáticas de grau 1 ao 4. (GUYTHER, 2016).

O baço é o órgão intra-abdominal que mais se lesa no traumatismo fechado e devido a seu tamanho e localização na cavidade abdominal, o fígado é lesado com grande frequência em traumatismos fechados e penetrantes (TOWNSEND, et al. 2014). Desta maneira, a abordagem das lesões de hepáticas e esplênicas nos grandes centros de trauma torna-se um assunto de alta relevância.

Historicamente o trauma de baço era tratado através de condutas cirúrgicas, até que em 1968 o controle não operatório de lesões em pediatria foi descrito pela primeira vez, passando a ter destaque a partir da década de 1980, em pacientes pediátricos com estabilidade hemodinâmica, e pouco depois, os mesmos princípios passaram a ser aplicados à paciente com traumas hepáticos e combinados de vísceras parenquimatosas abdominais com mesma taxa de sucesso (STEIN, 2006; PEREIRA JÚNIOR, 2007). 
Em um grande estudo multicêntrico realizado nos Estados Unidos, foi identificado que pacientes com idade superior a 55 anos tem uma maior probabilidade de ter complicações associadas à conduta não operatória devido a falta de reservas fisiológicas (STEIN, 2006; PEREIRA JÚNIOR, 2007; HARBRECT et al, 2001) o que ressalta o destaque de tal conduta entre pacientes jovens e, especialmente, os atendidos na pediatria.

Após observar os critérios de exclusão, relativos ou absolutos, de pacientes para a conduta não operatória, que incluem presença de outras lesões intraabdominais que necessitem de intervenção cirúrgica, idade superior a 55 anos de idade, presença de TCE e presença de instabilidade hemodinâmica que não responde a ressuscitação com fluidos, a conduta é adotada como tratamento preferencial (STEIN, 2006; PEREIRA JÚNIOR, 2007). Esse procedimento baseiase desde simples observação, com monitoração do hematócrito sérico, até a realização de angiografia com ou sem embolização (TOWNSEND, et al. 2014; STEIN, 2006; PEREIRA JÚNIOR, 2007).

Baseado nisso, percebe-se que é de extrema importância identificar o prognóstico dos pacientes submetidos a conduta não operatória e as principais razões pelas quais essa terapia falhou e levou a necessidade da adoção de condutas tradicionais.

A mudança da rotina de tratamento operatório de escolha, para o tratamento não operatório no trauma abdominal fechado de órgãos sólidos é uma das mais notáveis alterações na abordagem do trauma das últimas duas décadas. (VELMAHOS, 2003).

O trauma de baço era tratado exclusivamente com esplenectomia total até a descrição dos primeiros casos de infecções fulminantes na década de 1950, a partir de então, iniciou o uso de técnicas cirúrgicas conservadoras, particularmente a esplenorrafia, como alternativa. 
Com a evolução e popularização dos métodos modernos de diagnóstico por imagem, a partir da década de 1980, os pacientes pediátricos com estabilidade hemodinâmica passaram a ser tratados de maneira conservadora (sem cirurgia), com inúmeras vantagens, além da preservação do órgão: menor tempo de internação, menor número de complicações intra-abdominais, menor necessidade de transfusão e menores custos hospitalares.

Pouco tempo depois, os mesmos princípios foram utilizados para os pacientes pediátricos com trauma hepático e com traumas combinados de vísceras parenquimatosas abdominais com sucesso similar. (HURTUK, 2006).

Estes estudos encorajaram os cirurgiões a aplicar a mesma abordagem não operatória para os pacientes adultos.

Em contraste com a alteração de conduta na abordagem do trauma hepático e esplênico, o tratamento recomendado para o trauma renal tem sido não operatório há várias décadas. Diferentemente da hemorragia intraperitoneal do trauma de fígado e baço, a localização retroperitoneal dos rins auxilia no tamponamento da hemorragia em caso de lesão e o rico suprimento sangüíneo pode promover a adequada cicatrização após lesões parenquimatosas.3

A conduta conservadora deve ser adotada somente após o adequado estadiamento da lesão. $O$ objetivo de tal conduta é permitir um tratamento seguro ao paciente, evitando-se uma cirurgia desnecessária, muitas vezes, com ressecção parcial ou total do órgão, bem como todos os riscos inerentes ao procedimento cirúrgico e anestésico.

\section{OBJETIVOS}

\section{Objetivo geral}


Analisar os dados epidemiológicos dos pacientes pediátricos, com idade entre 0 a 16 anos, que sofreram trauma abdominal contuso, com lesão de baço e/ou fígado, e que foram atendidos no Pronto Socorro do Hospital de Base-DF (HBDF), no período entre 2012 e 2016.

\section{Objetivo específico}

- Descrever o perfil das crianças e adolescentes atendidos no Pronto Socorro HBDF com trauma abdominal fechado.

- Descrever as principais causas do trauma abdominal contuso com lesão de baço ou fígado em pacientes pediátricos.

- Analisar possíveis preditores de falha de tratamento expectante no trauma abdominal contuso

- Relacionar comorbidades com a falha de tratamento expectante no trauma abdominal contuso

- Determinar os fatores que levam a necessidade de intervenção cirúrgica.

- Determinar os fatores que levam a necessidade de cuidados intensivos.

- Determinar a taxa de sucesso da conduta expectante (não cirúrgica) desses pacientes.

\section{JUSTIFICATIVA}

O grande número de trauma abdominal contuso em pediatria e o impacto na morbimortalidade requerem dos centros especializados em trauma, manejo eficaz, redução de riscos e aumento da taxa de sobrevida. Nesse contexto, as condutas não-cirúrgicas têm grande importância nos cenários de trauma, devido ao seu grande potencial para cicatrização de órgãos parenquimatosos (fígado, baço, rins) e os menores índices de complicações associadas aos atos cirúrgicos.

A importância deste estudo está em analisar o perfil do paciente e de indicadores clínicos e laboratoriais, oferecendo dados que evidenciem as vantagens para a 
adoção da terapia não-operatória na lesão de baço e fígado em pacientes pediátricos vítimas de trauma abdominal contuso. Essa escolha terapêutica, evitaria as complicações associadas à conduta operatória e permitiria um menor tempo de permanência do paciente na unidade de saúde evitando complicações associadas à internação. E caso seja evidenciada alta taxa de sucesso e bom prognóstico, geraria menos gastos ao sistema de saúde, o que tem grande relevância devido à atual crise do sistema de saúde público brasileiro. Além disso, destaca-se a importância da atualização de bibliografia disponível sobre as condutas expectantes no tratamento de lesões de vísceras parenquimatosas de graus mais graves (IV e V), visto que os dados disponíveis sobre essa questão ainda são controvérsias.

\section{FUNDAMENTAÇÃO TEÓRICA}

O atendimento ao paciente politraumatizado depende da ação de profissionais de várias especialidades médicas, começando pelo adequado atendimento na cena do acidente, transporte rápido e seguro (por via terrestre ou aérea), uma avaliação consistente, ressuscitação volêmica, acurado diagnóstico e adequado tratamento intra-hospitalar. Os traumas podem variar desde escoriações superficiais, sem nenhum risco de vida, até grandes contusões ou ferimentos graves que causam a morte instantânea da vítima. Assim, o conhecimento do nível de gravidade de um paciente traumatizado é fator decisivo para orientar não apenas a conduta e o nível de complexidade hospitalar exigido, como também para aferir a qualidade do atendimento prestado.

Os índices de trauma são sistemas de pontuação criados para avaliar as alterações fisiológicas, a gravidade das lesões anatômicas e a probabilidade de sobrevida dos pacientes politraumatizados. (COIMBRA, 1997).

A análise dos índices de trauma permite, ainda, a apreciação de vários outros objetivos, são eles: 
- Quantificação das lesões anatômicas

- Cálculo da probabilidade de sobrevida

- Triagem de pacientes politraumatizados para centros de trauma

- Pesquisa clínica

- Avaliação de resultados institucionais

- Controle de qualidade

- Epidemiologia

- Campanha de prevenção de violência

- Pagamento de despesas médico-hospitalares

Os fatores que aumentam a taxa de mortalidade são:

-Atrasos no diagnóstico (após 24 horas da admissão).

-Erros no diagnóstico (lesões despercebidas devido a inadequado exame físico ou má interpretação de exames diagnósticos complementares).

-Erros de julgamento (decisões diagnósticas ou terapêuticas feitas contrariamente aos dados disponíveis).

-Erros de técnica (que ocorrem durante a realização de procedimentos diagnósticos ou terapêuticos).

As mortes no paciente politraumatizado, são classificadas em:

- Inevitáveis (lesão anatômica ou combinação de lesões consideradas de sobrevivência impossível, mesmo com tratamento adequado e a tempo, geralmente, correspondem a uma probabilidade de sobrevida, de acordo com os índices de trauma, menor que 25\%);

- Potencialmente salváveis (lesão anatômica ou combinação de lesões consideradas muito graves, mas com sobrevivência possível dentro de condições ótimas de atendimento; geralmente, a probabilidade de sobrevida encontra-se entre 25 e $50 \%)$; 
- Francamente evitáveis (lesão anatômica ou combinação de lesões consideradas de sobrevida possível, com probabilidade de sobrevida maior que 50\%). (SHACKFORD,1987)

A avaliação do Revisited Trauma Score (RTS), e para complementar a avaliação da gravidade dos pacientes politraumatizados, o índice, o Injury Severity Score (ISS), que, em associação ao RTS, forma a classificação denominada TRISS, a qual permite estabelecer a probabilidade de sobrevida do paciente, permite avaliar a qualidade do serviço e fazer comparação entre eles.

\section{REVISITED TRAUMA SCORE (RTS)}

O RTS, apresentado na tabela 1, é um índice classificado como fisiológico, por levar em consideração os parâmetros das funções vitais do paciente. É proveniente da avaliação do estado neurológico pela Escala de Coma de Glasgow - E.C.G., da pressão arterial sistólica (PAS) e da frequência respiratória (FR). As variáveis variam de 4 (normal) a 0.

\begin{tabular}{|c|c|c|c|c|c|}
\hline $\begin{array}{c}\text { ESCALA DE } \\
\text { COMA DE } \\
\text { GLASGOW }\end{array}$ & VALOR & $\begin{array}{c}\text { PRESSÃO } \\
\text { SISTÓLICA } \\
\text { (MMHG) }\end{array}$ & VALOR & $\begin{array}{c}\text { FREQUÊNCIA } \\
\text { RESPIRATÓRIA } \\
\text { (IPM) }\end{array}$ & VALOR \\
\hline $13-15$ & 4 & $>89$ & 4 & $10-29$ & 4 \\
\hline $9-12$ & 3 & $76-89$ & 3 & $>29$ & 3 \\
\hline $6-8$ & 2 & $50-75$ & 2 & $6-9$ & 2 \\
\hline $4-5$ & 1 & $1-49$ & 1 & $1-5$ & 1 \\
\hline 3 & 0 & 0 & 0 & 0 & 0 \\
\hline
\end{tabular}

Tabela 1: Revised Trauma Score (RTS)

PROBABILIDADE DE SOBREVIDA PARA RTS DE NÚMEROS INTEIROS 
É um índice largamente utilizado na triagem pré hospitalar, onde é chamado de tRTS, demonstrado na tabela 2, sendo seus valores simplesmente somados e, de acordo com o valor total, o paciente é transportado para determinado centro de trauma, previamente classificado, de acordo com a capacidade dos seus recursos diagnósticos, terapêuticos e humanos.

Para a avaliação intra-hospitalar, os valores das variáveis devem ser ponderados e somados:

\section{RTS = 0,9368 x E.C.G.v + 0,7326 x PASv + 0,2908 x FRv}

(onde v é o valor (de 0 a 4) correspondente as variáveis medidas na admissão do paciente).

\begin{tabular}{|c|c|}
\hline RTS & $\begin{array}{c}\text { Probabilidade de } \\
\text { sobrevida (PS) }\end{array}$ \\
\hline 8 & 0,988 \\
\hline 7 & 0,969 \\
\hline 6 & 0,919 \\
\hline 5 & 0,807 \\
\hline 4 & 0,605 \\
\hline 3 & 0,361 \\
\hline 2 & 0,172 \\
\hline 1 & 0,071 \\
\hline 0 & 0,027 \\
\hline
\end{tabular}

Tabela 2: Probabilidade de sobrevida para RTS

\section{INJURY SEVERITY SCORE (ISS)}


O ISS é um índice de gravidade, classificado como anatômico, por levar em consideração as lesões provocadas nos vários segmentos do corpo. (BAKER , 1974). A gravidade das lesões anatômicas é determinada através do exame físico, testes radiológicos, cirurgia e autópsia. (BAKER, 1976).

As lesões são classificadas em:

1) leve

2) moderada

3) grave, sem risco iminente de morte

4) grave, com risco iminente de morte

5) crítica, de sobrevida duvidosa

6) quase sempre fatal.

O ISS é calculado após a classificação dos índices mais graves de cada uma das seis (06) regiões, escolhendo-se os três (03) valores das AIS mais altos em segmentos corpóreos diferentes e realizando- se a soma dos quadrados desses índices. Desta maneira, o ISS pode variar de 1 a 75 pontos. Qualquer paciente com uma lesão AIS - 6 , tem um ISS = 75, por definição. O ISS correlaciona-se mais com a mortalidade tardia, enquanto que o RTS correlaciona-se melhor com a mortalidade precoce.

\section{TRAUMA AND INJURY SEVERITY SCORE (TRISS)}

Este é um índice que procura associar o índice fisiológico (RTS) ao índice anatômico (ISS), levando em consideração, ainda, a idade e o mecanismo de trauma, se contuso ou penetrante (CHAMPION, 1996)

O TRISS é o método utilizado pelo Colégio Americano de Cirurgiões e tem fundamental importância na análise retrospectiva da probabilidade de sobrevida, sendo utilizado no MTOS. (BOYD,1987) A análise do TRISS permitirá avaliar a 
qualidade do serviço prestado na instituição, bem como compará-la com a de outros centros de trauma. (BOYD,1987)

\section{TRATAMENTO NÃO OPERATÓRIO}

Os benefícios da terapia não-operatória (TNO) são menores gastos, menor tempo de internação necessária, redução da necessidade de transfusão sanguínea e evita a necessidade de laparotomia. A laparotomia, por si, conta com complicações como os riscos da anestesia, infecções da ferida, hérnias incisionais e lesões iatrogênicas (STEIN, 2006). Além disso, o tratamento cirúrgico da lesão esplênica leva desde enfraquecimento do sistema imune até o desenvolvimento de sepse fulminante pós-esplenectomia (TOWNSEND, et al. 2014). Desta forma, o tratamento não-operatório de tais lesões torna-se cada vez mais fundamental na área de atendimento a pacientes vítimas de trauma abdominal.

A conduta conservadora não operatória tornou-se o tratamento de escolha em pacientes vítimas de trauma contuso de órgãos parenquimatosos abdominais que apresentam estabilidade hemodinâmica (Sartorelli, 2000).

Recente estudo retrospectivo realizado em um Centro Hospitalar de alta complexidade de Oman (Arábia Saudita) publicado em 2013 avaliou a taxa de sucesso da conduta expectante (do inglês, Nonoperative Menagemnent - NOM) em pacientes vítimas de trauma abdominal por 10 anos (janeiro de 2001 à dezembro de 2011). O estudo selecionou 1071 pacientes para realizar o protocolo NOM do total de 5400 politraumatizados. Os critérios de exclusão usados no estudo foram: presença de instabilidade hemodinâmica (sinais de hemorragia, instabilidade persistente e não resposta as medidas de ressuscitação inicial ou lesão eminente de intestino). Todos os pacientes estáveis hemodinamicamente foram tratados conservadoramente. (RAZA et al., 2013). 
Nesse estudo o protocoloco NOM incluiu a avaliação dos sinais vitais, pulso, pressão arterial, temperatura, débito urinário, hemograma de 12 horas. 0 seguimento dos pacientes incluiu ultrassonografia abdominal ou TC, se os níveis de hemoglobina diminuíssem apesar da transfusão de 3 bolsas de sangue, se houvesse sinais de distensão abdominal progressiva, sinais de infecção, vômitos, hematúria ou taquipnéia. Essa conduta obteve sucesso em 963 pacientes (89.91\%) do total dos 1071 pacientes selecionados, sendo que em 108 pacientes ocorreram sinais de hemorragia, evidências tardias de perfuração de víscera oca ou infecção intra-abdominal o que exigiu laparotomia e portanto eles foram agrupados na categoria de falha da conduta NOM. O grupo que foi submetido à cirurgias tiveram uma taxa de admissão na UTI de $57 \%$, com um longo período de internação (23,31 dias) e maior morbidade (16\%) em comparação ao grupo submetido à conduta expectante que tiveram uma taxa de admissão na UTI de $24 \%$, com duração de estadia de 10,23 dias e morbidade $<1 \%$. O estudo concluiu, ainda, que o protocolo NOM pode ser usado também em pacientes com trauma abdominal de múltiplos órgãos, desde que ocorra um íntimo monitoramento hemodinâmico, garantindo altas taxas de sucesso terapêutico. (RAZA et al. 2013).

O uso da tomografia computadorizada (TC) nos pacientes com trauma abdominal contuso propicia o tratamento conservador não operatório das lesões de órgãos parenquimatosos, diminuindo a necessidade de cirurgia exploradora. A tendência atual em direção ao tratamento conservador não operatório de muitas lesões do fígado, baço e rins é devido, em parte, à capacidade da TC não apenas de definir a presença da lesão e a sua extensão, mas também de excluir outras lesões significantes, evitando cirurgias desnecessárias. (Wolfman, 1991).

O sucesso da abordagem conservadora não operatório para lesões de órgãos parenquimatosos em casos de trauma abdominal contuso e para casos selecionados de ferimentos por arma branca aceitos na maioria dos grandes centros de trauma, encorajou a conduta conservadora não operatória em pacientes com ferimentos penetrantes por arma de fogo. Atualmente dois 
argumentos utilizados para justificar o tratamento conservador não operatório, para ferimentos penetrantes abdominais, tanto por arma branca, quanto por arma de fogo, tem sido a significante morbidade, os custos associados à laparotomia não terapêutica (RENZ,1996) e o significante potencial para cicatrização de órgãos parenquimatosos (fígado, baço e rins) traumatizados como observado no tratamento de lesões graves desses órgãos no trauma contuso.( Moudouni, 2001).

\section{CRITÉRIOS DE SELEÇÃO DOS PACIENTES PARA O TRATAMENTO NÃO OPERATÓRIO}

Os critérios utilizados para selecionar os pacientes para a conduta conservadora não operatória é a condição hemodinâmica desses pacientes (que incluem a reposição volêmica mínima, ausência de significante traumatismo cranioencefálico, ausência de lesões intra-abdominais hemorragia ou requerer intervenção concomitantes, idade menor que 55 anos e ausência de outras lesões que possam influenciar a cirúrgica.

O TNO não é recomendado se a instituição for incapaz de acompanhar o paciente de forma seriada, incluindo tomografia computadorizada (TC), suporte intensivo, equipe de trauma e disponibilidade de uma laparotomia de emergência nas 24 horas do dia. Este método de tratamento não é totalmente seguro e sua incidência de falha varia entre $2 \%$ e $22 \%$. A presença de múltiplas lesões, volumoso hemoperitônio, idade maior que 55 anos e um ISS alto são fatores importantes associados à falha do TNO. (FERNANDES, 2013).

A experiência obtida em vários estudos realizados com pacientes pediátricos permite tentar tal tratamento em pacientes adultos com múltiplas lesões sem aumento da morbidade e mortalidade comparado com pacientes com lesão isolada de órgão parenquimatoso abdominal. (Sartorelli, 2000)

Nos anos iniciais o TNO só era indicado em doentes conscientes, mas, recentemente, tem sido indicado também para doentes com trauma 
craniencefálico, sob ventilação mecânica, e nesses casos a atenção da equipe tem que ser muito maior para uma eventual conversão para o tratamento cirúrgico. Há relatos de que TNO de lesões contusas do baço, claramente, tornou-se o padrão de atendimento em trauma pediátrico e que em 75 a $93 \%$ das lesões esplênicas em crianças tratadas desta forma pode ser observado sucesso terapêutico. (FERNANDES, 2013)

A presença de múltiplas lesões de órgãos parenquimatosos abdominais tem sido uma contra-indicação relativa para 0 tratamento não operatório. (SARTORELLI,2000)

\section{ESTADIAMENTO DAS LESÕES PARENQUIMATOSAS ABDOMINAIS}

O Comitê da "Organ Injury Scaling" (OIS) foi criado em 1987 dentro da Associação Americana de Cirurgia do Trauma para classificar o estadiamento e atualizar periodicamente os graus de lesões dos diversos órgãos. Sua classificação tem sido aceita mundialmente, estabelece cinco graus de lesões em ordem crescente de gravidade, como nas tabelas 3 e 4 . Tal classificação é utilizada para o estadiamento das lesões através da realização dos exames radiológicos ou pelo achado intraoperatório no momento da exploração cirúrgica.

\section{CRITÉRIOS DE SUCESSO E FALHA NO TRATAMENTO NÃO OPERATÓRIO}

O tratamento não operatório consiste de observação rigorosa intra-hospitalar, não necessariamente em leito de unidade de terapia intensiva (mas idealmente), repouso relativo no leito, seguimento do hematócrito, hidratação endovenosa abundante, jejum por 24 a 48 horas, ou seja, até uma melhor definição da não 
necessidade cirúrgica. (Mattheus, 1995)

\begin{tabular}{|c|c|c|}
\hline Grade & & Injury Description \\
\hline \multirow[t]{2}{*}{ I } & Haematoma & Subcapsular, $<10 \%$ surface area \\
\hline & Laceration & Capsular tear, $<1 \mathrm{~cm}$ parenchymal depth \\
\hline \multirow[t]{2}{*}{ II } & Haematoma & $\begin{array}{l}\text { Subcapsular, } 10-50 \% \text { surface area } \\
\text { Intraparenchymal, }<5 \mathrm{~cm} \text { diameter }\end{array}$ \\
\hline & Laceration & $1-3 \mathrm{~cm}$ parenchymal depth not involving a parenchymal vessel \\
\hline \multirow[t]{2}{*}{ III } & Haematoma & $\begin{array}{l}\text { Subcapsular, }>50 \% \text { surface area or expanding. } \\
\text { Ruptured subcapsular or parenchymal haematoma. } \\
\text { Intraparencymal haematoma }>5 \mathrm{~cm}\end{array}$ \\
\hline & Laceration & $>3 \mathrm{~cm}$ parenchymal depth or involving trabecular vessels \\
\hline IV & Laceration & $\begin{array}{l}\text { Laceration of segmental or hilar vessels producing major devascularization ( }>25 \% \text { of } \\
\text { spleen) }\end{array}$ \\
\hline \multirow[t]{2}{*}{$\mathrm{V}$} & Laceration & Completely shattered spleen \\
\hline & Vascular & Hilar vascular injury which devascularized spleen \\
\hline
\end{tabular}

Tabela 3: Estadiamento de lesão esplênica da OIS - revisão de 1994 (Moore et al.)

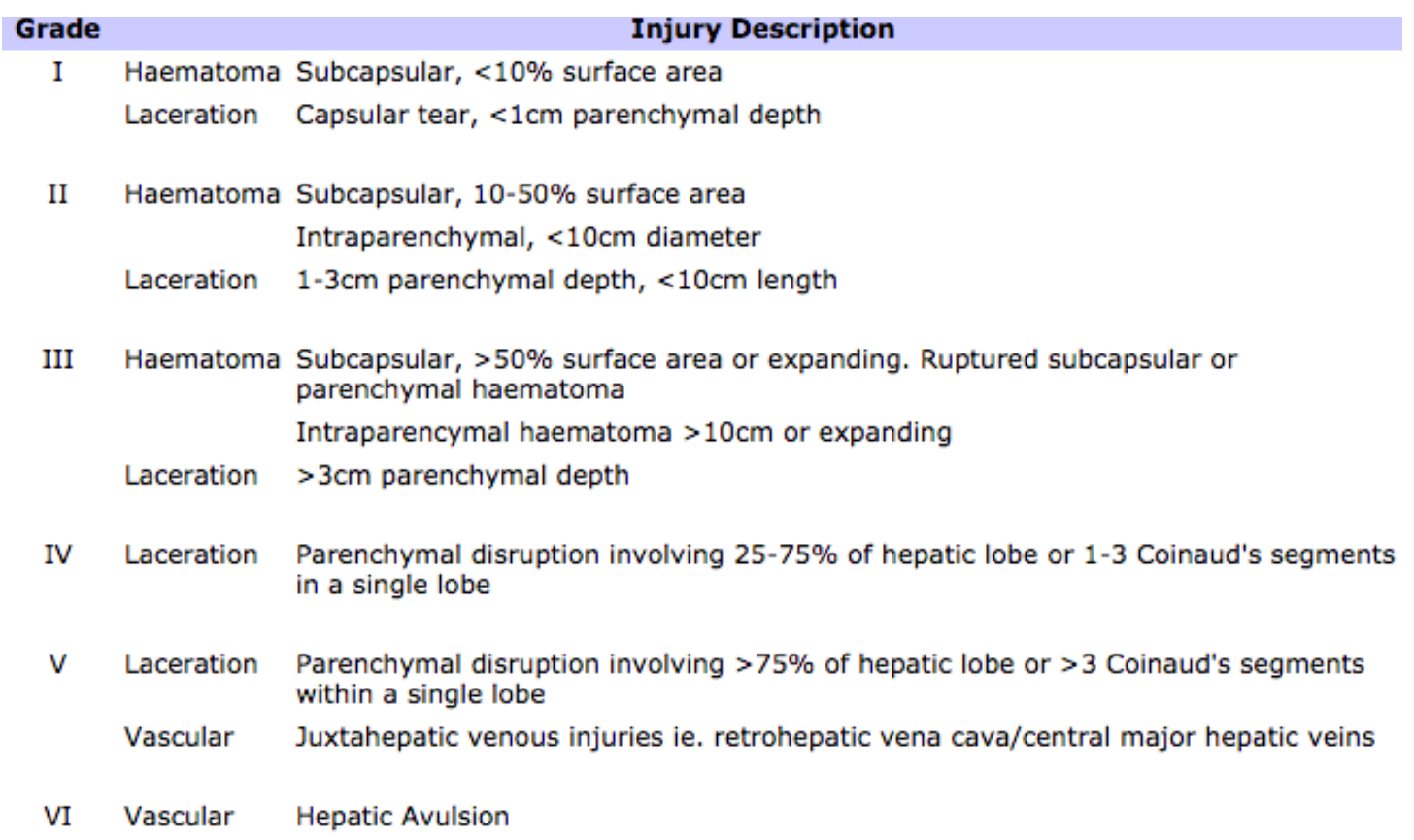

Tabela 4: Estadiamento de lesão hepática da OIS - revisão de 1994 (Moore et al.)

\section{CRITÉRIOS DE SUCESSO E FALHA NO TRATAMENTO NÃO OPERATÓRIO}


O tratamento não operatório consiste de observação rigorosa intra-hospitalar, não necessariamente em leito de unidade de terapia intensiva (mas idealmente), repouso relativo no leito, seguimento do hematócrito, hidratação endovenosa abundante, jejum por 24 a 48 horas, ou seja, até uma melhor definição da não necessidade cirúrgica. (Mattheus, 1995)

Os preditores de falha do tratamento não operatório são a presença de hipotensão na admissão, o alto grau de lesão e a necessidade de transfusão sangüínea. Um estudo recente mostrou que na presença de lesão renal ou esplênica com Focused Abdominal Sonography for Trauma (FAST) positivo, uma estimativa de líquido livre no CT maior do que $300 \mathrm{ml}$ e necessidade de hemotransfusão, o risco de falha no tratamento não operatório é de $96 \%$, enquanto que na ausência destes fatores, o risco de falha é de apenas $2 \%$. (VELMAHOS, 2003).

A presença destes fatores de risco indica a internação do paciente em terapia intensiva. Este estudo também mostrou que o tratamento não operatório das lesões esplênicas, particularmente aquelas de grau 3 ou mais, são mais propensas à falhas do que as lesões do rim ou fígado. (VELMAHOS ,2003).

A seguir, serão discutidos, individualmente, a abordagem dos traumas esplênico e hepático.

\section{TRAUMA ESPLÊNICO}

As lesões do baço ocorrem mais frequentemente associadas ao trauma contuso em acidentes automobilísticos, quedas e agressões físicas. Podem ocorrer de forma isolada ou associadas à outras lesões intra-abdominais.

O baço é um dos órgãos intra-abdominais mais freqüentemente lesados no trauma abdominal contuso. O dogma de que o baço não é essencial à vida perpetuou-se ao longo dos séculos, fazendo com que as esplenectomias totais 
fossem realizadas como primeira opção para o tratamento de lesões esplênicas. Entretanto, a incidência de fenômenos infecciosos após esplenectomias e a descoberta de que o baço é um importante órgão que atua na defesa orgânica fez com que novas medidas de abordagem das lesões esplênicas fossem consideradas. (KING,1952) (PIMPL, 1989).

Quando apropriadamente aplicado em lesões esplênicas, o tratamento não operatório (TNO) tem sido descrito com taxas de sucesso em 60 a $98 \%$ dos casos. (Peitzman,2000) (PachteR,1998).

Alguns autores relataram o tratamento não-operatório em crianças. A partir de então esta medida passou a ser adotada também em adultos, porém com menor freqüência. Somente na última década passou a ser amplamente utilizada tanto em crianças quanto em adultos. (BARONE,1999) (DAVIS,1998) (BRASEL,1998) (GROSS, 1999).

A ultrassonografia abdominal é um método complementar por imagem com alta sensibilidade para se diagnosticar líquido livre na cavidade peritoneal. (BENYA,2000) (MCKENNEY,1998).

A quantidade de líquido deve ser estimada pois esse dado é importante para orientar quanto à gravidade da lesão. O exame ultra-sonográfico não fornece dados sobre as características do líquido para afirmar se é sangue, urina ou conteúdo intestinal. É necessário orientar-se em parâmetros clínicos para descartar lesões associadas de vísceras ôcas intra-abdominais.

Quando este exame não foi o suficiente para a indicação de laparotomia completou-se a propedêutica com tomografia abdominal contrastada. A tomografia abdominal contrastada é um exame com alta especificidade para 0 diagnóstico de lesões de órgãos parenquimatosos. (BENYA E,1995) (EMERY, 1999). 
A presença de alteração vascular no baço, observada à tomografia abdominal, tem sido correlacionada com a falência do tratamento não-operatório. Esse achado consiste de uma coleção intraparenquimatosa hiperdensa de contraste (blush vascular) circunscrita pelo parênquima adjacente. No ato operatório podese confirmar que se trata de hematoma progressivo devido à hemorragia ativa no interior do baço. Muitas vezes, estes hematomas evoluem para ruptura. Alguns autores preconizam o tratamento operatório em vigência destes achados tomográficos. Outros indicam arteriografia esplênica com embolização arterial seletiva destes falsos aneurismas, com o intuito de melhorar o resultado do tratamento não-operatório.( SHANMUGANATHAN, 2000).

Em um estudo com grande casuística, as lesões penetrantes foram tratadas sem cirurgia em $62,4 \%$ dos casos e nos traumas contusos em $85,6 \%$ dos casos. A taxa de tratamento não operatório em pacientes com mais de 18 anos de idade com lesões esplênicas foi de $81,8 \%$, enquanto em menores de 18 anos de idade foi de 91,8\%. (HURTUK,2006).

Pacientes submetidos à cirurgia antes de 12 horas da admissão hospitalar geralmente são considerados como tratamento cirúrgico, enquanto que pacientes submetidos a cirurgia depois de 12 horas ou mais da admissão, são considerados como falha do tratamento conservador.

O tratamento conservador no trauma esplênico consiste na simples observação clínica ou angiografia, com ou sem embolização. Assim, podemos ter diferentes estratégias no tratamento conservador (STEIN, 2006):

- Observação: o período de observação de um paciente com trauma esplênico deve ser feito em ambiente monitorizado ou de cuidados intensivos. O paciente deve ser mantido em jejum e com avaliações seriados do hematócrito.

- Angiografia: a angiografia é uma terapia adjuvante nas lesões esplênicas que tem sido extensivamente investigada. 
A taxa de complicações de uma laparotomia não terapêutica pode chegar a 15\%. As complicações de uma esplenectomia, incluem: um aumento do risco de infecções e enfraquecimento do sistema imune secundário à asplenia. (STEIN, 2006).

A única contra-indicação absoluta do tratamento não operatório é o paciente com instabilidade hemodinâmica que não responde à reposição de fluidos. (STEIN, 2006).

Outras contra-indicações que são debatidas na literatura são: altos graus de lesão na TC, extravasamento ativo de contraste na TC, FAST positivo, hemoperitônio volumoso, ISS>25 e necessidade de transfusão de sangue. (MOORE, 1989).

Estudos mais recentes sugerem que os pacientes com lesão neurológica associada podem ser tratados conservadoramente com sucesso, inclusive havendo uma possibilidade de melhor prognóstico, por evitar a morbidade da anestesia e da laparotomia. (STEIN, 2006).

O seguimento com TC de abdome, geralmente, não é feito em lesões de baixo grau. Geralmente, a repetição do exame é feita de acordo com a sintomatologia clínica ou em lesões de grau avançado após 24 a 72 horas.

As falhas do tratamento não operatório podem resultar de hemorragias significativas e, subsequente, hipotensão e choque circulatório, que devem ser corrigidas com esplenectomia, ao invés de se tentar uma esplenorrafia.

As taxas de falha são bastante variáveis. Uma revisão recente demonstrou taxa de sucesso de $98 \%$ em crianças e $83 \%$ em adultos com lesões de grau I a III. (STEIN, 2006). 
Uma revisão retrospectiva foi publicada em 2000 com análise de 1488 pacientes com lesão esplênica. Trinta e oito por cento foi manejado com cirurgia. E, o restante foi manejado conservadoramente. A taxa de falha no tratamento conservador foi de $10,8 \%$. De acordo com o grau da lesão, as taxas de falhas foram de $4,8 \%$ no grau I, 9,5\% no grau II, $16,9 \%$ no grau III, $33,3 \%$ no grau IV e $75 \%$ no grau V. (PEITZMAN, 2000).

\section{TRAUMA HEPÁTICO}

O tratamento não-operatório (TNO) é basicamente influenciado pela condição hemodinâmica do paciente, o grau de lesão hepática, a presença de lesões abdominais associadas e alterações neurológicas. Os pacientes com trauma abdominal fechado com lesões concomitantes no fígado e no baço têm maior necessidade de transfusão sanguínea, maior mortalidade e maior taxa de falha do TNO. (ZAGO et. al, 2012).

O mecanismo de trauma mais frequente é o contuso devido a acidentes de transporte, seguido de agressões físicas. (HURTUK, 2006).

Os acidentes automobilísticos são o principal mecanismo de trauma hepático contuso. Pachter et al., em um estudo multicêntrico, encontraram que $72 \%$ dos 404 pacientes com trauma hepático foram vítimas de acidentes automobilísticos. (PACHTER, 1996).

Outro estudo com 136 pacientes demonstrou que os acidentes automobilísticos foram responsáveis por $84 \%$ dos pacientes com trauma hepático fechado, seguido por atropelamento (7\%), espancamento em $5 \%$ e acidentes com motocicleta em 2\%. (CROCE, 1995).

Von Bahten et al. demonstraram em sua série que $46,5 \%$ dos traumas hepáticos fechados foram causados por acidentes automobilísticos, 33,5\% por 
atropelamento e 9,5\% por espancamento. Em um estudo realizado na Suécia com 46 pacientes, os acidentes automobilísticos foram responsáveis por $43 \%$ dos casos de trauma hepático fechado.(NORRMAN,2009)

Na maioria dos estudos, a taxa de tratamento não operatório do trauma hepático durante o período do estudo variou de 74,6 a $87,1 \%$. (HURTUK , 2006)

Até 1995, o tratamento cirúrgico era o indicado para pacientes com trauma hepático fechado. A relutância dos cirurgiões em optar pelo TNO era associada com três preocupações principais: a de que a lesão hepática não pararia de sangrar até a intervenção cirúrgica, a de que a não drenagem de bile resultaria em fístulas biliares e infecção, e a possibilidade de negligenciar uma possível lesão associada. (MALHOTRA, 2000) (PACHTER,1996)( NORRMAN,2009) (KOZAR, 2005)

O conhecimento de que $86 \%$ das lesões hepáticas já pararam de sangrar no momento da cirurgia e o elevado número de laparotomias não terapêuticas fizeram do TNO o tratamento de escolha para os pacientes admitidos com estabilidade hemodinâmica. (VELMAHOS, 2003) (PACHTER, 1996) (CROCE,1995) (NORRMAN, 2009) (FELICIANO,1886).

O TNO associa-se à menor necessidade de hemotransfusão (Tabela 1), menores taxas de complicação e menor mortalidade para os pacientes com estabilidade hemodinâmica. (VELMAHOS, 2003) (PACHTER, 1996)( CROCE, 1995) (MEREDITH, 1994) (BYNOE, 1992).

A observação deve ser feita em um ambiente monitorizado, com exame abdominal seriado e hematócrito. Pacientes com lesões de grau maior em tratamento conservador estão sob maior risco de falha do tratamento. (STEIN, 2006). 
Além do benefício óbvio de evitar a morbidade de uma laparotomia, o tratamento conservador das lesões hepáticas tem demonstrado outros benefícios, incluindo menor necessidade de transfusão sangüínea, menor índice de complicações abdominais, menor tempo de permanência hospitalar e menor tempo de permanência em unidades de terapia intensiva. (STEIN, 2006).

Não existe um consenso para o seguimento com TC de abdome. Geralmente não é feito em lesões de baixo grau e a repetição do exame é realizada de acordo com a sintomatologia clínica ou em lesões de grau avançado após 7 a 10 horas. (STEIN, 2006) (BRICK, 1987).

Os fatores de risco para falhas do tratamento não operatório têm variado em diferentes estudos. O maior grau da lesão, pooling de contraste na tomografia, o tracking periportal de sangue, volume do hemoperitônio e a presença de múltiplas lesões de órgãos parenquimatosos. No entanto, vários trabalhos recentes, principalmente utilizando técnicas angiográficas divergem em relação aos fatores de risco de falha do tratamento não operatório. (HURTUK, 2006).

As complicações associadas ao tratamento não operatório do trauma hepático diferem daquelas atribuídas ao trauma esplênico devido às peculiaridades anatômicas e fisiológicas do fígado. Assim, podemos ter abscessos, fístulas biliares, biliomas e hemobilia. (STEIN, 2006) (BRICK, 1987).

Os abscessos e biliomas podem ser tratados com elevada taxa de sucesso por meio da drenagem percutânea. As lesões biliares podem ser tratadas com a utilização da drenagem biliar por meio da colangiografia endoscópica retrógrada. A hemobilia pode ser tratada com técnicas angiográficas. (SARTORELLI, 2000) (STEIN, 2006). 
Uma outra complicação atribuída ao tratamento das lesões hepáticas de grau elevado é o desenvolvimento da síndrome de compartimento abdominal. (STEIN, 2006).

Os estudos diferem quanto à freqüência destas complicações com variações de 5 a $12 \%$. As taxas mais relatadas nas várias publicações são: hemorragias (5\%), abscessos $(0,7 \%)$, lesões desapercebidas $(0,5 \%)$ e biliomas $(0,4 \%)$.(STEIN, 2006). (SARTORELLI, 2000) (BRICK, 1987)

\section{METODOLOGIA}

Este estudo baseia-se em uma pesquisa quantitativa, descritiva e retrospectiva com análise de prontuário eletrônico de pacientes pediátricos, entre 0 e 16 anos, atendidos no Pronto Socorro do Hospital de Base do Distrito Federal pela equipe da Cirurgia Pediátrica, entre os anos de 2012 e 2016, que sofreram trauma abdominal contuso com lesão hepática e/ou esplênica, e que foram submetidos a conduta expectante (não cirúrgica). Foram analisados todos os prontuários de pacientes atendidos no Pronto Socorro, os que foram encaminhados e internados na UTI Pediátrica e na enfermaria da Cirurgia Pediátrica, totalizando uma amostra de 312 prontuários, sendo que apenas 65 entraram nos critérios de inclusão.

Todos os dados levantados foram analisados por estatístico para o cruzamento dos mesmos e definição de porcentagens.

\section{Critérios de Inclusão:}

- Pacientes pediátricos de 0 a 16 anos de idade, vítimas de trauma abdominal fechado, com lesão de vísceras maciças, admitidos no Pronto Socorro do Hospital de Base DF, entre janeiro de 2012 a dezembro de 2016. 


\section{Critérios Exclusão}

- Paciente com trauma abdominal fechado com lesão de vísceras ocas.

- Paciente com desfecho letal nas primeiras 6 horas de internação hospitalar.

- Pacientes que receberam alta hospitalar com menos de 24 horas.

- Pacientes com dados no prontuário incompletos ou inadequadamente preenchidos.

Instrumental utilizado:

Foi elaborado um instrumento de coleta de dados (ANEXO 1) com as seguintes variáveis: ano de admissão, idade (classificada em recém-nascido, lactente, préescolar, escolar ou adolescente), sexo, meio de transporte para o HBDF (carro, ambulância, SAMU, helicóptero ou outros), víscera lesada, complicações, estadiamento da lesão (usando escala OIS e considerando, neste trabalho, as lesões graus 1 e 2 como leves, 3 e 4 como moderadas e 5 e 6 como graves, no caso de lesões hepáticas; e graus 1 e 2 como leves, 3 como moderadas e 4 e 5 como graves, no caso de lesões esplênicas), realização ou não de tratamento cirúrgico de baço ou fígado nas 12 primeiras horas, realização ou não de tratamento cirúrgico de baço ou fígado após as 12 primeiras horas, sinais vitais e exames na admissão (PAS, FR, pulso, Glasgow e hematócrito), realização ou não de FAST na admissão, se houve transfusão de concentrado de hemácias na admissão e de qual volume, se houve internação em UTI e por quanto tempo e a etiologia da lesão (colisão automobilística, acidente doméstico, atropelamento, queda de bicicleta ou outros).

A coleta de dados foi realizada no período de abril a maio de 2017.

A equipe de pesquisadores foi composta por Lucas Ferreira e Marcella Mateus (alunos de medicina do UniCEUB), bem como por Andréa Kairala, médica Intensivista Pediatra e orientadora da pesquisa. Os alunos foram responsáveis pela pesquisa, leitura e atualização bibliográfica, pela obtenção dos dados 
referentes aos pacientes, pela elaboração do relatório parcial, pela elaboração do relatório final e pela elaboração do artigo científico, sempre com orientação da Dra Andréa. A análise dos dados obtidos foi realizada por um estatístico.

Realizou-se análise dos dados por meio da estatística descritiva, com o objetivo de caracterizar a amostra quanto ao ano de internação, sexo, idade, cor, motivos da internação e todas as outras variáveis contidas no instrumento de coleta de dados.

A análise univariada foi descrita em valores absolutos, médias e percentuais (por meio de tabelas). Para a análise de associação entre as variáveis (bivariada), utilizou-se o teste Qui-Quadrado. Tal teste foi usado para descobrir se existe uma associação/relação estatisticamente significante entre as variáveis coletadas e relacionadas à falha da conduta expectante no trauma abdominal fechado. Para análise, foi adotado um nível de significância a considerado de 5\%; ou seja, se, ao realizar o teste, o resultado gerado possuir um valor abaixo de 0,05 , concluiuse que existe relação entre as variáveis. Como exemplo, se o $p$-valor resultar em valor de $0,001(<0,05)$ demonstra-se alta relação entre as variáveis estudadas.

Para a elaboração do artigo científico, um levantamento bibliográfico e revisão de literatura das pesquisas e assuntos mais recentes na área foi realizado, por meio de bases de dados automatizadas como MEDLINE, SCIELO, PUBMED, LILACS e SECRETARIA DA SAÚDE, utilizando como palavras-chave: Traumatismo abdominal fechado. Tratamento não operatório. Fígado; lesões. Baço; lesões. Pediatria. O período dos artigos pesquisados foi de 2000 a 2017, em língua inglesa, espanhola e portuguesa.

Por ser um estudo não intervencionista (sem intervenções clínicas), com presença de risco mínimo aos pacientes e familiares, foi proposta a dispensa do TCLE (em anexo), a qual foi aceita. O estudo respeitou as regulamentações nacionais relativas à privacidade e à investigação médica. Em nenhum momento da 
execução e exposição desse estudo houve identificação dos pacientes ou entrevista de pais ou responsáveis.

O projeto foi aprovado pelo Comitê de Ética em Pesquisa do Centro Universitário de Brasília, CAAE 62412216.8.0000.0023.

\section{RESULTADOS}

Foram analisados inicialmente 312 prontuários; 65 foram selecionados de acordo com os critérios de inclusão.

Com relação aos anos analisados, 13 pacientes foram analisados no ano de 2012; 14 no ano de 2013; 24 no ano de 2014; 7 no ano de 2015 e 7 no ano de 2016 (Gráfico1).

Com relação ao gênero, 43 dos pacientes eram do sexo masculino e 22 do sexo feminino (Gráfico 2). Sobre a faixa etária, 6 pacientes eram lactentes, 22 pré escolares, 32 escolares e 5 adolescentes. A tabela 5 mostra a relação entre entre sexo e faixa etária dos pacientes. 


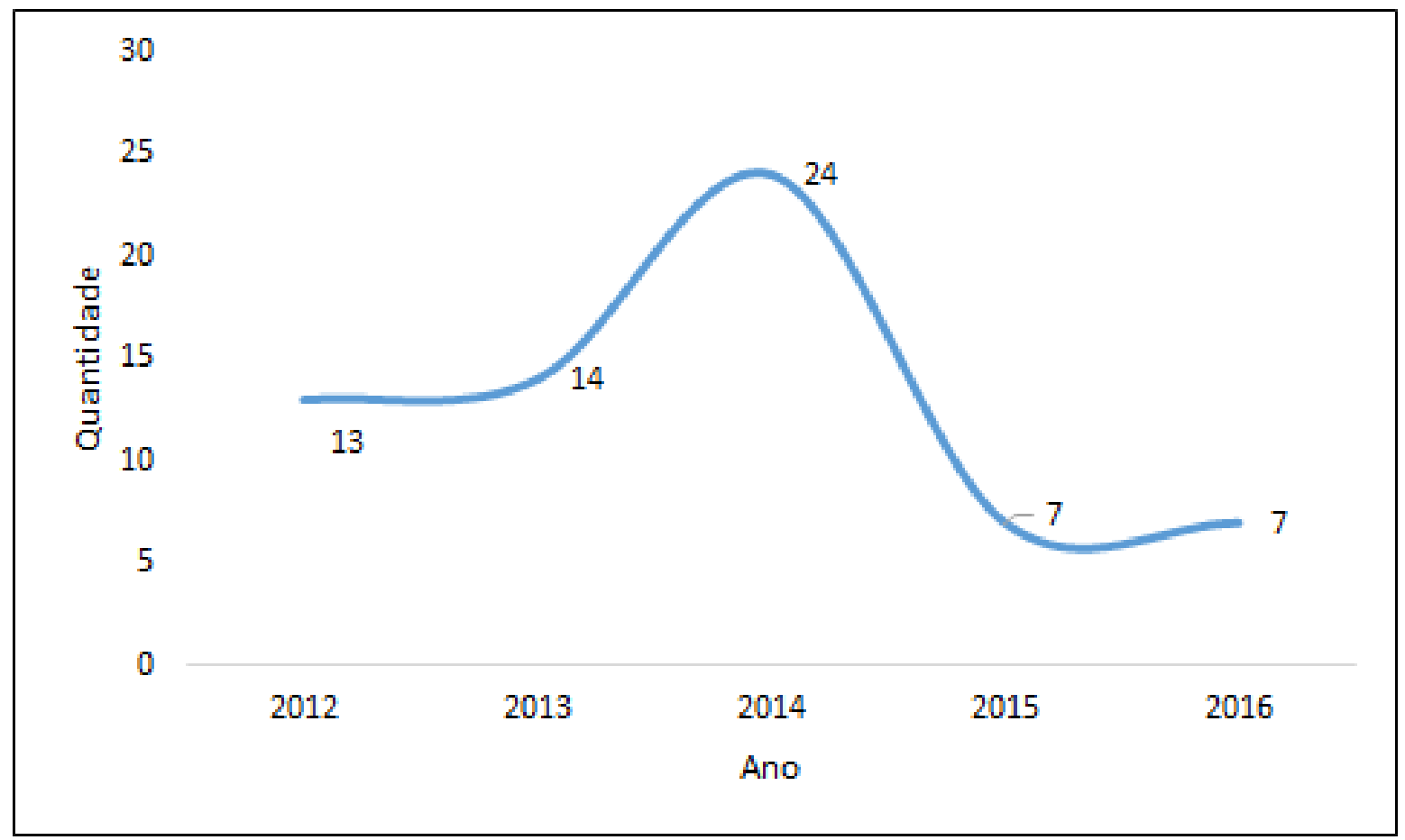

Gráfico 1: Distribuição do número de casos por período.

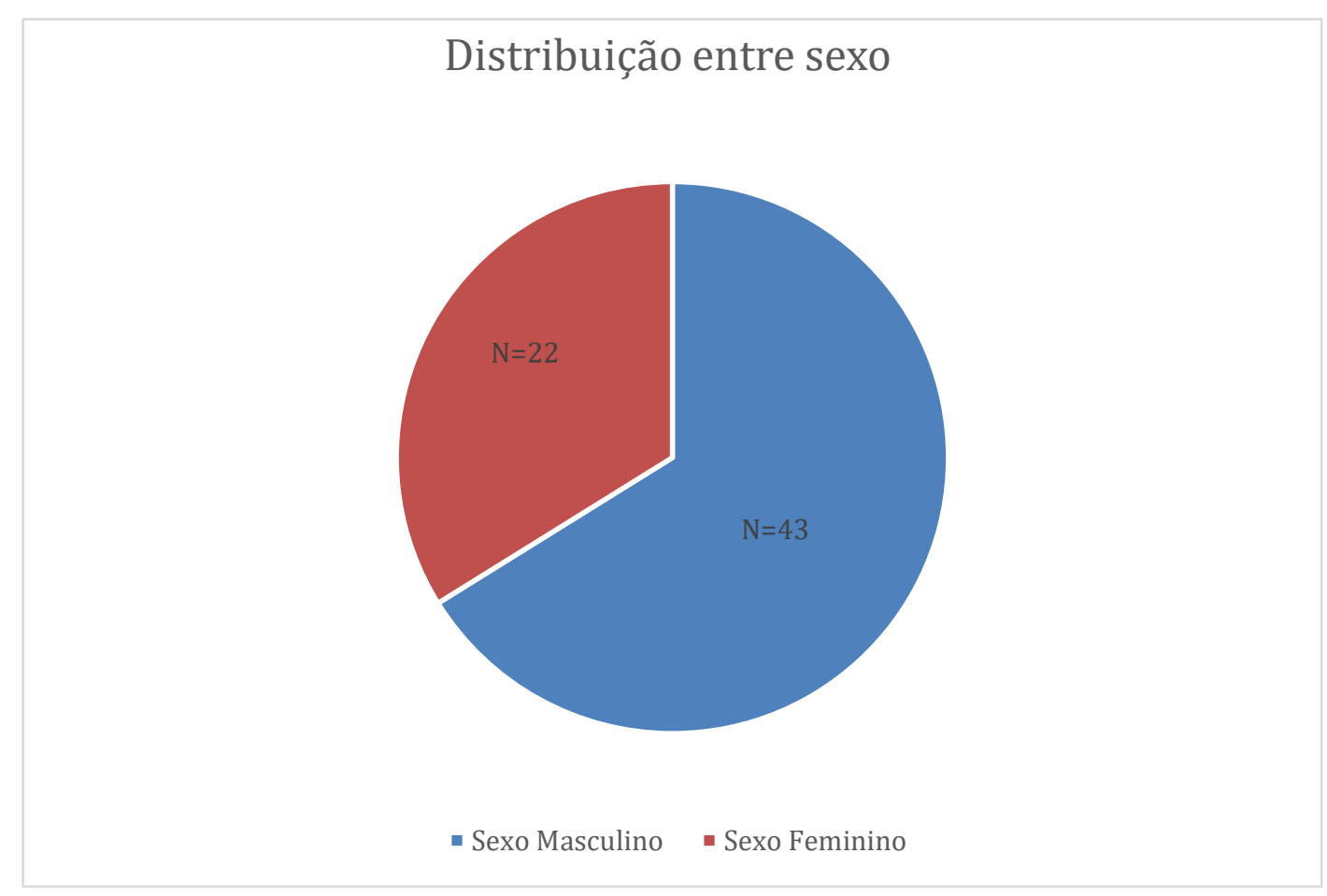

Gráfico 2: Distribuição do número de casos por sexo 


\begin{tabular}{|c|c|c|c|c|c|c|}
\hline \multirow{3}{*}{$\begin{array}{l}\text { Faixa } \\
\text { Etária }\end{array}$} & \multicolumn{4}{|c|}{ Sexo } & \multirow{2}{*}{\multicolumn{2}{|c|}{ Total }} \\
\hline & \multicolumn{2}{|c|}{ Feminino } & \multicolumn{2}{|c|}{ Masculino } & & \\
\hline & № & $\%$ & № & $\%$ & № & $\%$ \\
\hline Lactente & 1 & 4,5 & 5 & 11,6 & 6 & 9,2 \\
\hline Pré Escolar & 11 & 50,0 & 11 & 25,6 & 22 & 33,8 \\
\hline Escolar & 8 & 36,4 & 24 & 55,9 & 32 & 49,2 \\
\hline Adolescente & 2 & 9,1 & 3 & 6,9 & 5 & 7,8 \\
\hline Total & 22 & 100 & 43 & 100 & 65 & 100 \\
\hline
\end{tabular}

Tabela 5: Relação entre sexo e faixa etária dos pacientes

Ao analisar os dados referentes as vísceras lesadas, notou-se que 33 pacientes apresentavam lesão apenas de fígado, 27 apenas de baço e 5 lesaram ambas as vísceras (Gráfico 3).

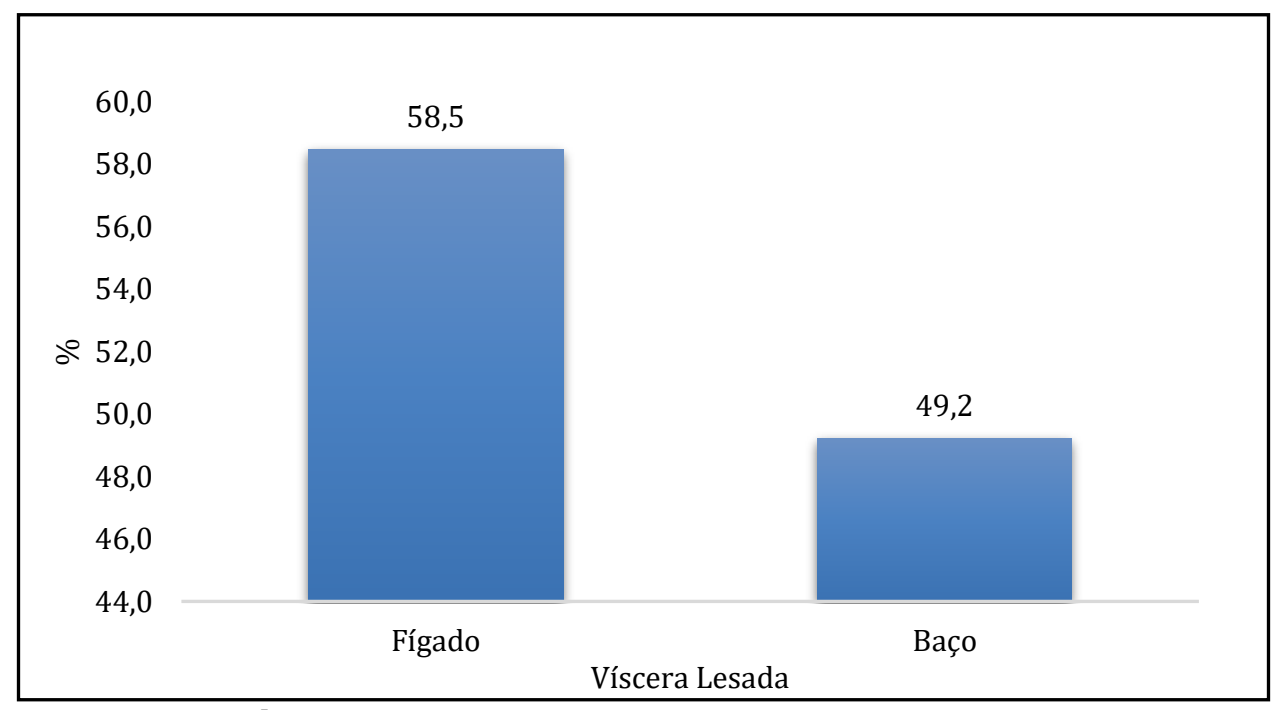

Gráfico 3: Distribuição das vísceras lesadas

A análise dos dados referentes a etiologia do trauma demonstrou que colisões automobilísticas foram responsáveis por $29 \%$, atropelamentos por $21 \%$, 
capotamentos por $11 \%$, quedas de bicicleta por $11 \%$, acidentes domésticos por $10 \%$ e etiologias não citadas na ficha de coleta de dados por 18\% (Gráfico 4).

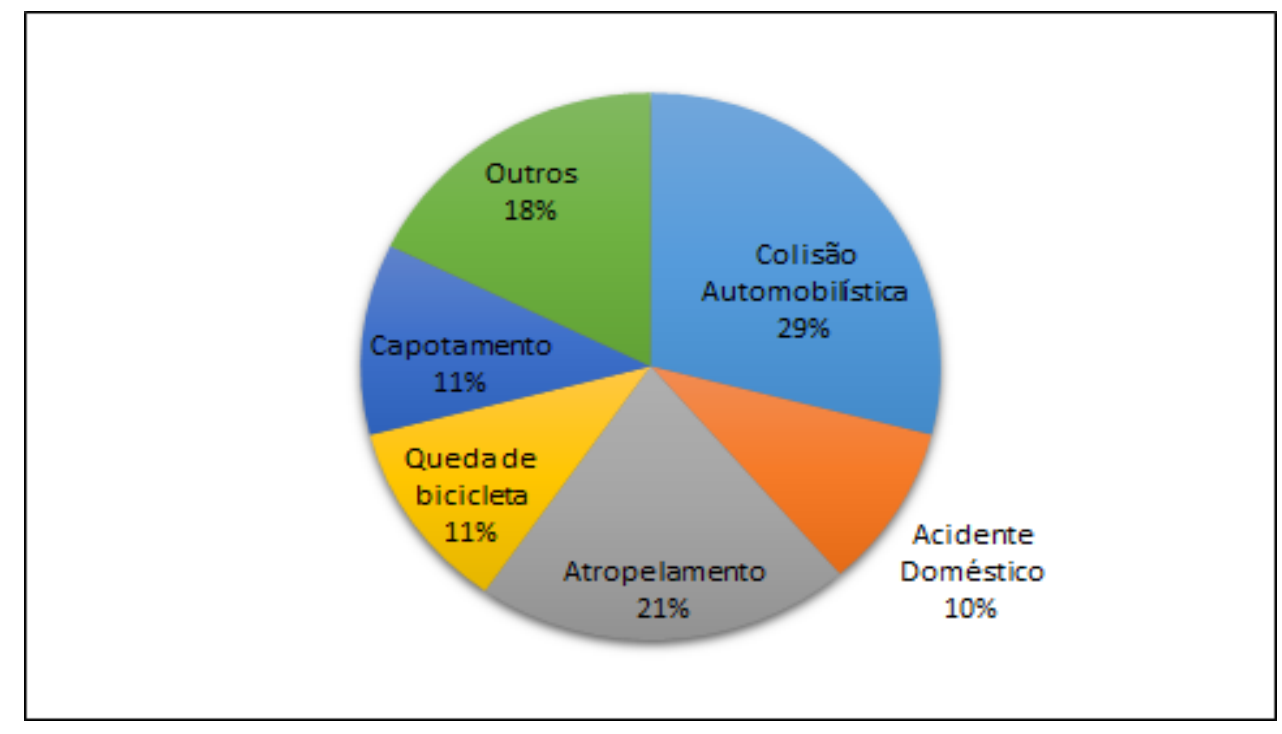

Gráfico 4: Distribuição de etiologia das lesões

Quando comparado o estadiamento da lesão e o tipo de víscera lesada observouse que há relação entre as duas variáveis analisadas. O p-valor $=0,048$ o que evidencia haver forte relação. O gráfico 5 demonstra este comportamento tendo 0 Fígado uma predominância de lesões do tipo leve enquanto no baço há predominância de lesões do tipo Moderado.

Em relação ao meio de transporte utilizado para levar o paciente até o local do atendimento inicial, no caso o HBDF, 22 foram transportados de ambulância, 19 pelo SAMU, 5 de helicóptero e 19 não continham esta informação no prontuário (Gráfico 6).

Quanto a análise da relação entre o tipo de transporte e o estadiamento da lesão, não há relação entre essas variáveis. 


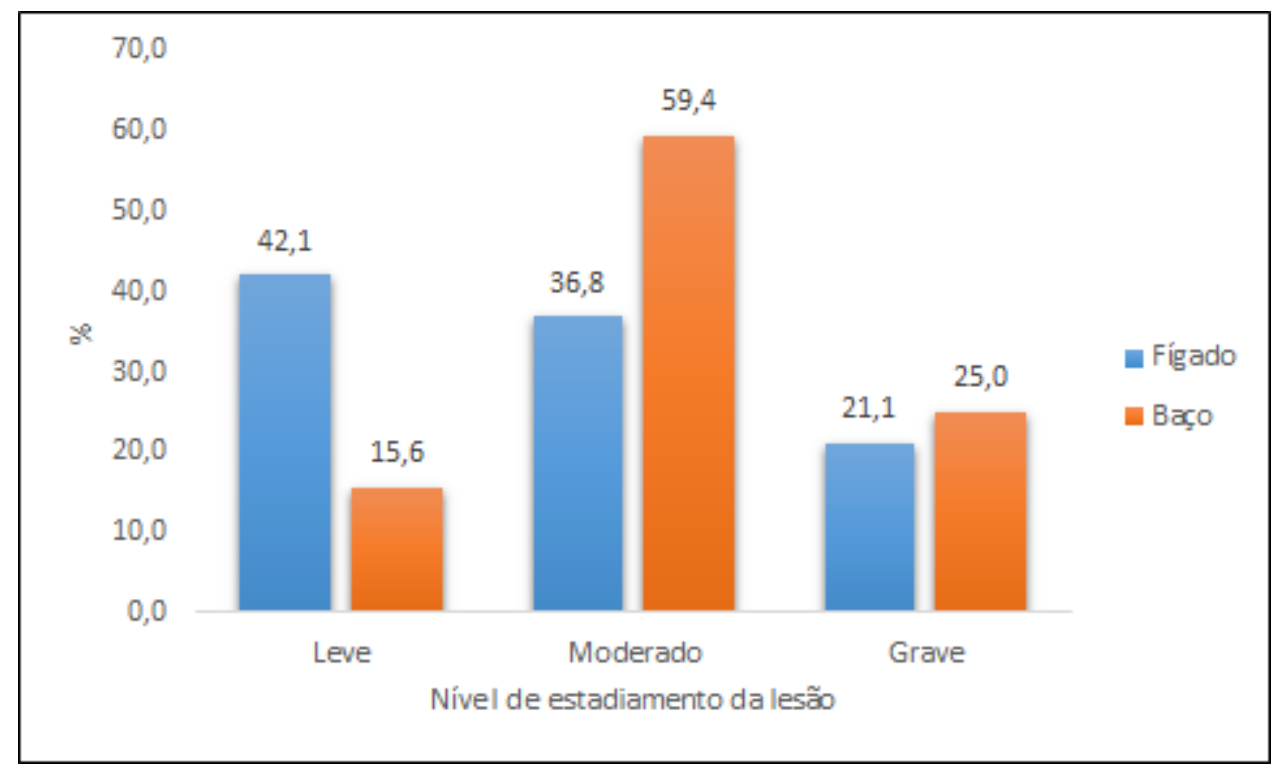

Gráfico 5: Relação entre estadiamento da lesão e tipo de víscera lesada

Quando o tipo de transporte escolhido foi ambulância, percebe-se 54,5\% tiveram lesão hepática leve seguido de lesão hepática moderada. (27,3\%). Já ao analisar os pacientes com lesão esplênica transportados via ambulância observou-se que $53,8 \%$ tinham lesões moderadas seguidos de lesões esplênicas graves que corresponderam a $30,8 \%$.

No caso dos pacientes transportados pelo SAMU ou Helicóptero não se observou nenhum padrão de comportamento.

Ao analisar o estadiamento de acordo com a gravidade da lesão e sua relação com causa do acidente (colisão automobilística, acidente doméstico, atropelamento queda de bicicleta) não se percebeu relação estatística (Tabela 6).

Quando analisado a existência de relação entre víscera lesada e idade do paciente observou-se que que o baço foi mais frequentemente lesado entre os escolares $(68,8 \%)$ e o fígado mais lesado entre os pré-escolares, correspondendo a $50 \%$ (Tabela 7 ). 


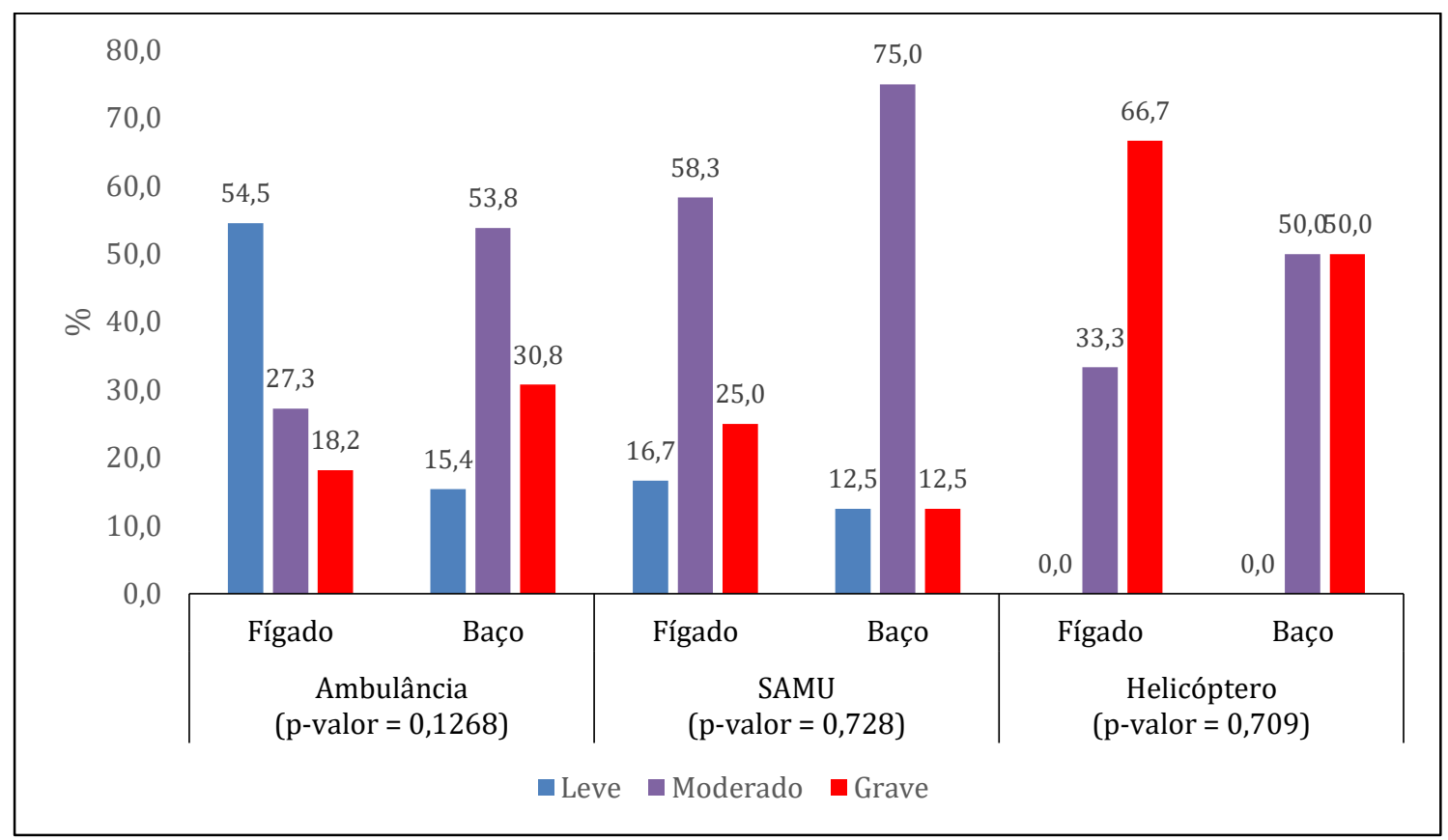

Gráfico 6: Relação entre o tipo de transporte e o estadiamento da lesão

Já ao relacionar dados referentes ao sexo do paciente e a víscera lesada foi evidenciado que não há relação entre tais variáveis, ou seja, a distribuição entre ambos os sexos é a mesma.

\begin{tabular}{|c|c|c|c|c|c|c|}
\hline \multirow{2}{*}{$\begin{array}{l}\text { Etiologia de } \\
\text { lesão }\end{array}$} & \multirow{2}{*}{$\begin{array}{c}\text { Local } \\
\text { da } \\
\text { lesão }\end{array}$} & \multicolumn{3}{|c|}{ Estadiamento } & \multirow[b]{2}{*}{ Total } & \multirow{2}{*}{ p-valor } \\
\hline & & Leve & Moderado & Grave & & \\
\hline Colisão & Fígado & 35,7 & 50,0 & 14,3 & 100 & \multirow{2}{*}{0,9495} \\
\hline Automobilística & Baço & 42,9 & 42,9 & 14,3 & 100 & \\
\hline Acidente & Fígado & 0,0 & 66,7 & 33,3 & 100 & \multirow{2}{*}{0,3494} \\
\hline Doméstico & Baço & 25,0 & 75,0 & 0,0 & 100 & \\
\hline \multirow{2}{*}{ Atropelamento } & Fígado & 28,6 & 28,6 & 42,9 & 100 & \multirow{2}{*}{0,2858} \\
\hline & Baço & 0,0 & 66,7 & 33,3 & 100 & \\
\hline & Fígado & 50,0 & 50,0 & 0,0 & 100 & 0,6456 \\
\hline
\end{tabular}




\begin{tabular}{c|l|r|r|r|r|l}
\hline $\begin{array}{c}\text { Queda de } \\
\text { bicicleta }\end{array}$ & Baço & 20,0 & 60,0 & 20,0 & 100 & \\
\hline \multirow{2}{*}{ Capotamento } & Fígado & 50,0 & 16,7 & 33,3 & 100 & \multirow{2}{*}{0,5135} \\
& Baço & 50,0 & 50,0 & 0,0 & 100 & \\
\hline \multirow{2}{*}{ Outros } & Fígado & 75,0 & 25,0 & 0,0 & 100 & \multirow{2}{*}{0,0260} \\
& Baço & 0,0 & 85,7 & 14,3 & 100 & \\
\hline
\end{tabular}

Tabela 6: Distribuição proporcional da causa do acidente e relação com gravidade da lesão.

\begin{tabular}{|c|c|c|c|c|c|c|}
\hline \multirow{3}{*}{ Variável } & \multirow{3}{*}{ Categoria } & \multicolumn{4}{|c|}{ Víscera lesada } & \multirow{3}{*}{ p-valor } \\
\hline & & \multicolumn{2}{|c|}{ Fígado } & \multicolumn{2}{|c|}{ Baço } & \\
\hline & & Qtde & $\%$ & Qtde & $\%$ & \\
\hline \multirow{3}{*}{ Sexo } & Feminino & 13 & 35,1 & 10 & 32,3 & \multirow{3}{*}{0,804} \\
\hline & Masculino & 24 & 64,9 & 21 & 67,7 & \\
\hline & Total & 37 & 100 & 31 & 100 & \\
\hline \multirow{5}{*}{ Idade } & Lactente & 6 & 15,8 & 0 & 0,0 & \multirow{5}{*}{0,002} \\
\hline & Pré Escolar & 19 & 50,0 & 8 & 25,0 & \\
\hline & Escolar & 10 & 26,3 & 22 & 68,8 & \\
\hline & Adolescente & 3 & 7,9 & 2 & 6,3 & \\
\hline & Total & 38 & 100 & 32 & 100 & \\
\hline
\end{tabular}

Tabela 7: Distribuição do tipo de víscera lesada quanto ao sexo e faixa etária do paciente

A taxa de total de óbito entre os pacientes estudados foi de $\mathrm{N}=4 ; 6,2 \%$ (Tabela 8) Dentre os 65 pacientes do estudo 39(60\%) necessitaram de internação na unidade de terapia intensiva, por tempo variado entre 2 e 211 dias, com uma média de 15,9 dias. 


\begin{tabular}{c|c|c|c}
\hline Variável & Categoria & Quantidade & $\%$ \\
\hline \multirow{2}{*}{$\begin{array}{c}\text { Admissão } \\
\text { em UTI }\end{array}$} & Sim & 39 & 60,0 \\
\cline { 2 - 4 } & Não & 26 & 40,0 \\
\cline { 2 - 4 } & Total & 65 & 100,0 \\
\hline \multirow{3}{*}{ Óbito } & Sim & 4 & 6,2 \\
\cline { 2 - 4 } & Não & 61 & 93,8 \\
\cline { 2 - 4 } & Total & 65 & 100,0 \\
\hline Escala de & ECG $=<8$ & 9 & 17,6 \\
\cline { 2 - 4 } Coma de & ECG $=>9$ & 42 & 82,4 \\
\cline { 2 - 4 } Glasgow & Total & 51 & 100,0 \\
\hline
\end{tabular}

Tabela 8: informações sobre possíveis sinais de gravidade

Ao considerar a existência de comorbidades associadas ao trauma abdominal (TCE, lesão pulmonar e outros) e internação em UTI, observou-se que esses pacientes foram mais frequentemente admitidos em UTI ( $p$-valor=0,00224), o que mostra uma relação bastante significativa entre tais variáveis (Tabela 9).

\begin{tabular}{|c|c|c|c|c|c|}
\hline \multirow{3}{*}{$\begin{array}{c}\text { Tipo de } \\
\text { Comorbidades } \\
\text { relacionadas ao } \\
\text { acidente }\end{array}$} & \multicolumn{4}{|c|}{ Internação na UTI } & \multirow{3}{*}{ p-valor } \\
\hline & \multicolumn{2}{|c|}{ Sim } & \multicolumn{2}{|c|}{ Não } & \\
\hline & Qtde & $\%$ & Qtde & $\%$ & \\
\hline Não houve & 12 & 30,8 & 20 & 76,9 & \\
\hline TCE & 13 & 33,3 & 1 & 3,8 & \\
\hline Lesão pulmonar & 9 & 23,1 & 3 & 11,5 & 0,00224 \\
\hline Outros & 5 & 12,8 & 2 & 7,7 & \\
\hline Total & 39 & 100 & 26 & 100 & \\
\hline
\end{tabular}

Tabela 9: Relação entre comorbidade e internação na UTI 
Com relação a intervenções cirúrgicas, $13,8 \%$ dos pacientes foram submetidos a abordagem cirúrgica inicial de fígado ou baço nas primeiras 12 horas de internação e 4,6\% após as 12 primeiras horas de internação (Tabela 10).

Dentre os pacientes submetidos a conduta expectante, $53,57 \%$ foram internados na UTI, com percentual de óbitos de $0 \%$, enquanto entre os que não foram acompanhados na UTI, o percentual de óbitos foi de 7,69\%.

A taxa de óbito entre os pacientes submetidos a conduta cirúrgica em algum momento foi de $20 \%$, enquanto entre os submetidos a conduta totalmente expectante foi de $3,64 \%$.

Ao comparar o estadiamento da lesão e o tempo decorrido entre admissão e abordagem cirúrgica obteve-se que nas lesões leves não houve abordagem cirúrgica em nenhum dos casos pesquisados. No tocante as lesões hepáticas de grau moderado e grave não houve relação entre lesão e intervalo entre admissão e tratamento cirúrgico. Já ao analisar as lesões esplênicas obteve-se pvalor $=0,039$ indicando que que há relação significativa entre tal lesão e tempo para abordagem cirúrgica.

Ao se analisar a relação entre pacientes submetidos a cirurgia em menos de $12 \mathrm{~h}$ de admissão e o valor do hematócrito, obteve-se p-valor significativo $(0,0156)$, havendo maior frequência dentre os casos com baixo hematócrito. Dos pacientes com hematócrito baixo $27,7 \%$ foram submetidos a abordagem cirúrgica nas primeiras $12 \mathrm{~h}$ de internação. Já dentre aqueles com hematócrito normal para idade apenas $5,26 \%$ foram submetidos a tratamento cirúrgico com menos de $12 \mathrm{~h}$ de internação. O que demonstra haver relação entre valor do hematócrito e tempo decorrido entre admissão e abordagem cirúrgica (Tabela11).

Já ao analisar pacientes submetidos a cirurgia após $12 \mathrm{~h}$ de internação observouse que tal fato não tem relação com o valor do hematócrito visto que o $p$-valor obtido foi de 0,185 . 


\begin{tabular}{|c|c|c|c|c|c|c|}
\hline \multirow[t]{2}{*}{$\begin{array}{c}\text { Local do } \\
\text { estadiamento } \\
\text { da lesão }\end{array}$} & \multirow[t]{2}{*}{ Grau } & \multicolumn{2}{|c|}{$\begin{array}{c}\text { Abordagem } \\
\text { cirurgica das } \\
\text { lesões em }<12 \mathrm{~h} \text { da } \\
\text { admissão }\end{array}$} & \multicolumn{2}{|c|}{$\begin{array}{c}\text { Abordagem } \\
\text { cirurgica das } \\
\text { lesões em }>12 \mathrm{~h} \text { da } \\
\text { admissão }\end{array}$} & \multirow[t]{2}{*}{ p-valor } \\
\hline & & Qtde & $\%$ & Qtde & $\%$ & \\
\hline \multirow{3}{*}{ Fígado } & Moderado & 3 & 100,0 & 2 & 66,7 & \multirow{3}{*}{0,2733} \\
\hline & Grave & 0 & 0,0 & 1 & 33,3 & \\
\hline & Total & 3 & 100,0 & 3 & 100,0 & \\
\hline \multirow{3}{*}{ Baço } & Moderado & 5 & 83,3 & 0 & 0,0 & \multirow{3}{*}{0,039} \\
\hline & Grave & 1 & 16,7 & 0 & 0,0 & \\
\hline & Total & 6 & 100,0 & 0 & 0,0 & \\
\hline
\end{tabular}

Tabela 10: Relação entre estadiamento da lesão e tempo decorrido entre admissão e abordagem cirúrgica

\begin{tabular}{|c|c|c|c|c|c|c|}
\hline \multirow{3}{*}{ Variável } & \multirow{3}{*}{ Categoria } & \multicolumn{4}{|c|}{ Valor de referência do Hematócrito } & \multirow{3}{*}{ p-valor } \\
\hline & & \multicolumn{2}{|c|}{$\begin{array}{c}\text { Baixo para a } \\
\text { idade }\end{array}$} & \multicolumn{2}{|c|}{ Normal } & \\
\hline & & № & $\%$ & № & $\%$ & \\
\hline Abordagem & Sim & 6 & 27,27 & 2 & 5,26 & \\
\hline cirurgica & Não & 16 & 72,73 & 36 & 94,74 & \\
\hline das lesões & Total & & & & & 0,0156 \\
\hline$e m<12 h$ da & & 22 & & 38 & & \\
\hline admissão & & & 100,00 & & 100,00 & \\
\hline Abordagem & Sim & 1 & 4,55 & 0 & 0,00 & \\
\hline cirurgica & Não & 21 & 95,45 & 38 & 100,00 & \\
\hline das lesões & Total & & & & & 0,185 \\
\hline$e m>12 h$ da & & 22 & & 38 & & \\
\hline admissão & & & 100,00 & & 100,00 & \\
\hline
\end{tabular}

Tabela 11: Relação entre hematócrito e tempo decorrido entre admissão e abordagem cirúrgica. 
Observou-se relação entre necessidade de transfusão sanguínea e admissão na UTI (p-valor=0,0124). Dos pacientes que realizaram transfusão, $85 \%$ foram admitidos em UTI. Porém quanto ao grupo que não realizou transfusão apenas $50 \%$ necessitaram de internação em unidade de tratamento intensivo (Tabela 12).

\begin{tabular}{|c|c|c|c|c|c|c|}
\hline \multirow{3}{*}{ Variável } & \multirow{3}{*}{ Categoria } & \multicolumn{4}{|c|}{ Realizou transfusão? } & \multirow{3}{*}{ p-valor } \\
\hline & & \multicolumn{2}{|c|}{ Sim } & \multicolumn{2}{|c|}{ Não } & \\
\hline & & Qtde & $\%$ & Qtde & $\%$ & \\
\hline \multirow{3}{*}{$\begin{array}{c}\text { Internação } \\
\text { na UTI }\end{array}$} & Sim & 17 & 85,0 & 14 & 50,0 & \multirow{3}{*}{0,0124} \\
\hline & Não & 3 & 15,0 & 14 & 50,0 & \\
\hline & Total & 20 & 100,0 & 28 & 100,0 & \\
\hline
\end{tabular}

Tabela 12: Relação entre transfusão e internação na UTI

A relação entre tempo decorrido da admissão e abordagem cirúrgica com a realização ou não de transfusão não é significativa. Porém percebe-se que os pacientes que não necessitaram de transfusão foram menos abordados cirurgicamente do que o grupo que recebeu hemoderivados (Tabela 13).

\begin{tabular}{c|c|c|c|c} 
Variável & Categoria & Realizou transfusão? & Total & p-valor \\
\hline
\end{tabular}




\begin{tabular}{|c|c|c|c|c|c|c|c|c|}
\hline & & \multicolumn{2}{|c|}{ Sim } & \multicolumn{2}{|c|}{ Não } & \multirow[b]{2}{*}{ № } & \multirow[b]{2}{*}{$\%$} & \\
\hline & & № & $\%$ & № & $\%$ & & & \\
\hline Abordagem & Sim & 5 & 25,0 & 2 & 7,1 & 7 & 14,6 & \\
\hline cirúrgica & Não & 15 & 75,0 & 26 & 92,9 & 41 & 85,4 & \\
\hline das lesões & Total & & & & & & & 008394 \\
\hline $\mathrm{em}<12 \mathrm{~h}$ & & 20 & & 28 & & & & \\
\hline $\mathrm{da}$ & & & & & & & & \\
\hline admissão & & & 100,0 & & 100,0 & 48 & 100,0 & \\
\hline Abordagem & Sim & 2 & 10,0 & 0 & 0,0 & 2 & 4,2 & \\
\hline cirúrgica & Não & 18 & 90,0 & 28 & 100,0 & 46 & 95,8 & \\
\hline das lesões & Total & & & & & & & 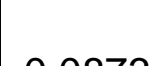 \\
\hline$e m>12 h$ & & & & & & & & $0,08 / 3$ \\
\hline $\mathrm{da}$ & & & & & & & & \\
\hline admissão & & 20 & 100,0 & 28 & 100,0 & 48 & 100,0 & \\
\hline
\end{tabular}

Tabela 13: Relação entre paciente que realizaram transfusão e tempo decorrido entre admissão e abordagem a cirúrgica

\section{DISCUSSÃO}

Dentre os pacientes avaliados neste estudo, houve um maior predomínio desse tipo de lesão em pacientes escolares e do sexo masculino; e também um predomínio das lesões hepáticas, porém a literatura não apresenta uma definição quanto a estes dados, uma vez que o espaço amostral varia entre os diversos estudos.

Nota-se que, assim como identificado por Guyther et al. em 2016, as colisões automobilísticas e os atropelamentos são as principais causas de trauma abdominal fechado com lesão de vísceras ocas. A queda de bicicleta, apresentou grande percentual de casos, devendo, portanto, ser valorizado, devido ao seu alto potencial lesivo. 
Evidenciou-se a maior probabilidade de lesões leves no fígado e moderadas no baço, porém, a etiologia da lesão não mostrou ter muita relevância no estadiamento, a não ser, no fato de que os mecanismos não citados na ficha de coleta de dados levam a maior tendência de lesões leves em fígado e moderadas em baço. Por fim, notou-se que o baço é mais lesado entre escolares e o fígado entre pré escolares e que o gênero não tem relação importante com a víscera lesada, sendo tais informações importantes para que a etiologia e a idade dos pacientes sirvam como preditores dos possíveis órgãos lesados e da gravidade destas lesões.

Apesar do maior percentual de transportes ao HBDF ser realizado por ambulância, não há relação estatística entre o estadiamento da lesão e o meio de transporte escolhido, o que relaciona-se ao fato da baixa relação entre etiologia e grau da lesão, uma vez que a inexistência desta relação faz com que o meio de transporte escolhido não possa ser o mais apropriado a gravidade da lesão já que esta análise só poderá ser realizada em ambiente intra-hospitalar e o meio de transporte é escolhido de acordo com a gravidade aparente do caso.

A análise do meio de transporte utilizado não está presente na bibliografia analisada, sendo algo inédito, e indicando a necessidade da criação de meios de análise rápida da gravidade da lesão em ambiente pré-hospitalar, permitindo a definição do meio de transporte mais adequado ao caso.

Os cuidados em terapia intensiva mostram-se essenciais na recuperação da maior parte dos pacientes, principalmente naqueles que apresentam alguma comorbidade, sendo o período de permanência na UTI muito variável e específica para cada caso.

Assim como descrito por Velhamos et. al. em 2003, a grande maioria dos pacientes teve abordagem totalmente expectante. A abordagem cirúrgica tardia apresentou alta relação com a realização de procedimento cirúrgico envolvendo 
baço ou fígado nas 12 primeiras horas após a internação e o percentual de óbitos entre os pacientes submetidos a abordagem cirúrgica foi muito maior, o que confirma um melhor desfecho da conduta expectante. Porém, o hematócrito baixo está fortemente relacionado a abordagem cirúrgica precoce e consequentemente a maior probabilidade de necessidade de abordagem cirúrgica tardia e desfecho com óbito, devendo então ser considerados como sinal de alerta para um pior prognóstico, fator preditivo da falha da terapia expectante e indicativo da necessidade de cuidados intensivos precoces, o que corrobora com a descrição de Zago et al. em 2012 de que a condição hemodinâmica do paciente é um dos principais fatores a influenciar o TNO.

Por fim, apesar da descrição de Velhamos et. al. em 2003, o estudo não evidenciou relação entre a necessidade de intervenção cirúrgica e a realização de transfusão sanguínea, o que pode ser justificado pelo pequeno espaço amostral da presente pesquisa, porém, foi evidenciada maior necessidade de cuidados intensivos, confirmando a maior gravidade destes pacientes.

\section{CONCLUSÃO}

O resultado do estudo está em concordância com a bibliografia levantada e confirma uma adoção da TNO como primeira escolha nos casos de trauma abdominal contuso e um melhor prognóstico aos pacientes submetidos a esta terapia.

A terapia intensiva apresenta-se como essencial no seguimento do TNO.

Ressalta-se a importância do correto preenchimento dos prontuários nos serviços de saúde, uma vez que diversos prontuários precisaram ser excluídos da pesquisa devido à falta de informações, o que prejudica a realização de pesquisas essenciais a área médica. 
A maior parte da literatura descreve o TNO em pacientes adultos, sendo então necessária a realização de mais estudos entre os pacientes pediátricos, permitindo assim a análise dos mesmos dados em outros espaços amostrais.

\section{REFERENCIAS BIBLIOGRÁFICAS}

BAKER SP; O'NEILL B; HADDON JR W \& LONG WB. The injury severity score: a method for describing patients with Multiple injuries and evaluating emergency care. J Trauma 14: 187 - 196, 1974.

BAKER SP \& O'NEILL B. The injury severity score: an update. J Trauma 16: 882$885,1976$.

Barone JE, Burns G, Svehlak SA, et al. - Managment of blunt splenic trauma in patients older than 55 years. Southern Connecticut Regional Trauma Quality Assurance Committee. J Trauma, 1999, 46(1):87-90.

Benya EC, Lim-Dunham JE, Landrum $O$, et al. - Abdominalsonography in examination of children with blunt abdominal trauma. AJR Am J Roentgenol, 2000, 174(6): 1613-1616.

Benya EC, Bulas DI, Eichelberger MR, et al. - Splenic injury from blunt abdominal trauma in children: follow-up evaluation with TC. Radiology, 1995, 195(3):685- 688.

BOYD CR; TOLSON MA \& COPES WS. Evaluating trauma care: the TRISS method. J Trauma 27: 370-378, 1987.

Bynoe RP, Bell RM, Miles WS, Close TP, Ross MA, Fine JG. Complications of nonoperative management of blunt hepatic injuries. J Trauma. 1992;32(3):308-14; discussion 314-5. 
Brasel KJ, Olson CJ, Stafford RE, et al. - Incidence and significance of free fluid on abdominal computed tomographic scan in blunt trauma. J Trauma., 1998, 44(5): 889-892.

Brick SH, Taylor GA, Potter BM, Eichelberger MR. Hepatic and splenic injury in children: role of CT in the decision for laparotomy. Radiology 1987; 165: 643-6.

CARVALHO, Fábio $\mathrm{H}$. et al. Fatores prognósticos relacionados à falha do tratamento não-operatório de lesões esplênicas no trauma abdominal fechado. Revista do Colégio Brasileiro de Cirurgiões, vol 36 n2, Rio de Janeiro. Mar/Apr. 2009.

CHAMPION HR; SACCO WJ \& COPES WS. Trauma Scoring. In: MOORE EE; MATTOX KL; FELICIANO DV, eds, Trauma, $3^{\mathrm{a}}$ eds, Norwalk, Connecticut/ San Mateo, Califórnia, Appleton \& Lange; cap. 5, p. 53-67, 1996

Croce MA, Fabian TC, Menke PG, Waddle-Smith L, Minard G, Kudsk KA, et al. Nonoperative management of blunt hepatic trauma is the treatment of choice for hemodynamically stable patients. Results of a prospective trial. Ann Surg. 1995;221(6):744- 53; discussion 753-5.

COIMBRA RSM; ANGLE N; SILVA SE; HOYT DB \& RASSLAN S. Índices de trauma: o que são e por que devem ser usados. Rev Col Bras Cir 24: 255-263, 1997

Davis KA, Fabian TC, Croce MA, et al. - Improved success in nonoperative management of blunt splenic injuries. Embolization of splenic artery pseudo aneurysms. J Trauma, 1998, 44(6):1008-1015. 
DEMETRIADES, Demetrios. Seletive Nonoperative Managment of Penetrating Abdominal Solid Organ Injuries. Annals of Surgery, vol. 4, № 4, 244: 620-628, Oct. 2006.

Emery $\mathrm{KH}$, Babcock DS, Borgman AS, et al. - Splenic injury diagnosed with CT: US follow-up and healing rate in children and adolescents. Radiology, 1999, 212(2):515-518.

Feliciano DV, Mattox KL, Jordan GL Jr, Burch JM, Bitondo CG, Cruse PA. Management of 1000 consecutive cases of hepatic trauma (1979-1984). Ann Surg. 1986;204(4):438-45.

Feliciano DV, Burch JM, Spjut-Patrianely V, Mattox KL, Jordan GL Jr. Abdominal gunshot wounds. An urban trauma center's experience with 300 consecutive patients. Ann Surg 1988; 208: 362-70.

FERNANDES, Thaís M. et al. Tratamento não operatório de lesão esplênica grau IV é seguro usando-se rígido protocolo. Revista do Colégio Brasileiro de Cirurgiões, vol.40 no4, Rio de Janeiro. July/Aug. 2013.

Gross M, Lynch F, Canty T, et al. - Management of pediatric liver injuries: a 13 year experience at a pediatric trauma center. J Pediatr Surg, 1999, 34(5):811-817.

GUYTHER, Jennifer E.; LINZER, Jeffrey F. Advances in Pediatric Abdominal Trauma: What's New in Assessment and Management. Trauma Reports, vol 17, no 5, sept/oct. 2016.

HARBRECT et al. Contribution of age and gender to outcome of blunt splenic injury in adults: a multicenter study of the Eastern Association for the Surgery of Trauma. J Trauma.; 51:887,895. 2001. 
Hurtuk M, Reed RL, Esposito TJ, Davis KA, Luchette FA. Trauma surgeons practice what they preach: the NTDB story on solid organ injury management. $J$ Trauma 2006, 61:243-55.

ILIAS Elias J.; KASSAB Paulo. Qual a conduta no trauma abdominal fechado?. Revista da Associação Médica Brasileira vol.53 ㄲo 3. São Paulo May/June, 2007.

Kozar RA, Moore JB, Niles SE, Holcomb JB, Moore EE, Cothren CC, et al. Complications of nonoperative management of high-grade blunt hepatic injuries. $\mathrm{J}$ Trauma. 2005;59(5):1066-71.

King $\mathrm{H}$, Shumacker Jr HB - Susceptibility to infection after splenectomy performed in infancy. Ann Surg, 1952, 136:239-242.

McKenney KL, Nuñez DB, McKenney MG, et al. - Sonography as the primary screening technique for blunt abdominal trauma: experience with 899 patients. AJR Am J Roentgenol, 1998, 170(4): 979-985

Malhotra AK, Fabian TC, Croce MA, Gavin TJ, Kudsk KA, Minard G, et al. Blunt hepatic injury: a paradigm shift from operative to nonoperative management in the 1990s. Ann Surg. 2000;231(6):804-13.

Mattheus LA, Spirnak JP. The nonoperative approach to major blunt renal trauma. Sem Urol 1995; 23: 77-82.

Meredith JW, Young JS, Bowling J, Roboussin D. Nonoperative management of blunt hepatic trauma: the exception or the rule? J Trauma. 1994;36(4):529-34; discussion 534-5.

Moore EE, Shackford SR, Pachter HL, McAninch JW, Browner BD, Champion HR et al. Organ injury scaling: spleen, liver and kidney. J Trauma 1989; 29: 1664-6. 
Moore EE; Cogbill TH; Jurkovich GJ; Shackford SR; Malangoni MA; Champion HR. Organ injury scaling: spleen and liver (1994 revision). J Trauma 1995; 38:323-4.

MOORE et al. Organ injury scaling: spleen and liver (1994 revision). J trauma. ; 38:323-324. 1995.

Moudouni SM, Patard JJ, Manunta A, Guiraud P, Guille F, Lobel B. A Conservative approach to major blunt renal lacerations with urinary extravasation and devitalized renal segments. BJU Int 2001; 87: 290-4.

Norrman G, Tingstedt B, Ekelund M, Andersson R. Non-operative management of blunt liver trauma: feasible and safe also in centres with a low trauma incidence. HPB. 2009;11(1):50-6

Pachter HL, Guth AA, Hofstetter SR, Spencer FC. Changing patterns in the management of splenic trauma: the impact of nonoperative management. Ann Surg. 1998;227(5):708-17; discussion 717-9.

Pachter HL, Knudson MM, Esrig B, Ross S, Hoyt D, Cogbill T, et al. Status of nonoperative management of blunt hepatic injuries in 1995: a multicenter experience with 404 patients. J Trauma. 1996;40(1):31-8.

Peitzman AB, Heil B, Rivera L, Federle MB, Harbrecht BG, Clancy KD, et al. Blunt splenic injury in adults: Multi-institutional Study of the Eastern Association for the Surgery of Trauma. J Trauma. 2000;49(2):177-87; discussion 187-9.

PEREIRA JÚNIOR, Gérson A. Tratamento não operatório do trauma de vísceras abdominais parenquimatosas. Medicina, Ribeirão Preto, 40 (4): 538-550, out./dez. 2007. 
PEREIRA JÚNIOR, Gerson A.; SCARPELINI S; BASILE-FILHO A \& ANDRADE JI. Trauma severity indices. Medicina, Ribeirão Preto, 32: 237-250, july/sept. 1999.

Pimpl W, Dapunt O, Kaindl H, et al. - Incidence of septic and thromboembolicrelated deaths after splenectomy in adults. Br J Surg, 1989, 76(5):517-521.

RAZA M, et al. Non operative management of abdominal trauma - a 10 years review. World Journal of Emergency Surgery, 8:14. 2013

Renz BM, Feliciano DV. The length of hospital stay after unnecessary laparotomy for trauma: a prospective study. J

Trauma 1996; 40: 187-90.

Sartorelli KH, Frumiento C, Rogers FB, Osler TM. Nonoperative management of hepatic, splenic, and renal injuries in adultswith multiple injuries. J Trauma 2000; 49: 56-62.

SHACKFORD SR; FRIDLUND PH; MCARDE M \& EASTMAN AB. Assuring quality in a trauma system - The medical audit committee: Composition, cost, and results. J Trauma 27:866-875, 1987

Shanmuganathan K, Mirvis SE, Boyd-Kranis R, et al. - Nonsurgical management of blunt splenic injury: use of CT criteria to select patients for splenic arteriography and potential endovascular therapy. Radiology, 2000, 217(1):75-82

STEIN, Deborah M. Nonoperative Management of Spleen and Liver Injuries. J Intensive Care Med, 21: 296-304, 2006.

TOWNSEND, Courtney M. et al. Sabiston Tratado de Cirurgia: A Base Biológica da Prática Cirúrgica Moderna. 19aㅡ ed. Rio de Janeiro: Elsevier, 2014. 
Velmahos GC, Toutouzas KG, Radin R, Chan L, Demetriades D. Nonoperative treatment of blunt injury to solid abdominalorgans. Arch Surg 2003, 138: 844-51.

Velmahos GC, Toutouzas K, Radin R, Chan L, Rhee P, Tillou A, et al. High success with nonoperative management of blunt hepatic trauma: the liver is a sturdy organ. Arch Surg. 2003;138(5):475- 80; discussion 480-1.

ZAGO, Thiago M. Trauma hepático contuso: comparação entre o tratamento cirúrgico e o não operatório. Revista do Colégio Brasileiro de Cirurgiões, Rio de Janeiro, vol. 39 no 4, jul/aug. 2012.

Wolfman NT, Bechtold RE, Scharling ES, Meredith JW. Blunt upper abdominal trauma: evaluation by CT. Am J Roentgenol 1992; 158: 493-501.

- Ano de admissão

\section{ANEXO 1 QUESTIONÁRIO}

(1) 2013

(2) 2014

(3) 2015

(4) 2016

Sinais vitais/exames na admissão ao hospital

- PAS :

- FR:

- Pulso:

- Escala de Glasgow: 


\section{- Idade}

(1) RN (até 27 dias de vida)

(2) Lactente (28 dias até 2 anos incompletos)

(3) Pré escolar ( 2 anos a 7 anos incompletos)

(4) Escolar (7 anos a 12 anos incompletos)

(5) Adolescente (12 a 18 anos)

\section{- $\underline{\text { Sexo }}$}

(1) Feminino

(2) Masculino

- Transporte para o HBDF:

(1) Carro

(2) Ambulância

(3) SAMU

(4) Helicóptero

(5) Outros

- Víscera lesada

(1) fígado

(2) baço

(3) Outra

- Complicações:

- Estadiamento da Lesão

(segundo o exame de imagem)

(1) Grau I

(2) Grau II

(3) Grau III

(4) Grau IV

(5) Grau V

Etiologia de lesão (mecanismo traumático):

(1) Acidente automobilístico (carro X carro)

(2) Trauma doméstico

(3) Outros

Comorbidades:

Internação na Uti: (1) sim (2)não

Motivo da não internação na UTI:

Tempo de Internação na UTI:

- Tratamento cirúrgico

(cirurgia antes das 12 horas de admissão= tratamento cirúrgico):

(1) sim (2) não (3) não informado

- Tratamento cirúrgico

(cirurgia depois das 12 horas de admissão= falha do tratamento conservador):

(1) sim (2) não (3) não informado 